\title{
An engineered bispecific human monoclonal antibody against SARS-CoV-2
}

\author{
Zhaohui Li ${ }^{1,2,13}$, Shihua Li1,13, Gen Zhang ${ }^{1,13}$, Weiyu Peng ${ }^{1,3,13}$, Zhen Chang4 ${ }^{4}$ Xue Zhang ${ }^{4}$, Zheng Fan', \\ Yan Chai', Feiran Wang ${ }^{1,5}$, Xin Zhao', Dedong Lij ${ }^{1,3}$, Rong Zhang ${ }^{1,6}$, Zhanlong He${ }^{7}$, Weiwei Zou ${ }^{4}$, \\ Ke $\mathrm{Xu}^{8}$, Wenwen $\mathrm{Lei}^{8}$, Peipei Liu ${ }^{8}$, Junfeng Hao ${ }^{9}$, Jingjing Zhang ${ }^{10}$, Litao Sun ${ }^{10}{ }^{10}$, Guizhen $\mathbf{W u}^{8}$, \\ Shuguang Tan ${ }^{1 凶}$, George Fu Gao ${ }^{1 凶}$, Feng Gao $\mathbb{1}^{11,12 \bowtie}$ and Yan Wu ${ }^{1,4}{ }^{凶}$
}

\begin{abstract}
The global severe acute respiratory syndrome coronavirus 2 (SARS-CoV-2) pandemic requires effective therapies against coronavirus disease 2019 (COVID-19), and neutralizing antibodies are a promising therapy. A noncompeting pair of human neutralizing antibodies (B38 and H4) blocking SARS-CoV-2 binding to its receptor, ACE2, have been described previously. Here, we develop bsAb15, a bispecific monoclonal antibody (bsAb) based on B38 and H4. bsAb15 has greater neutralizing efficiency than these parental antibodies, results in less selective pressure and retains neutralizing ability to most SARS-CoV-2 variants of concern (with more potent neutralizing activity against the Delta variant). We also selected for escape mutants of the two parental mAbs, a mAb cocktail and bsAb15, demonstrating that bsAb15 can efficiently neutralize all single-mAb escape mutants. Furthermore, prophylactic and therapeutic application of bsAb15 reduced the viral titer in infected nonhuman primates and human ACE2 transgenic mice. Therefore, this bsAb is a feasible and effective strategy to treat and prevent severe COVID-19.
\end{abstract}

A $s$ the COVID-19 pandemic continues, several antiviral therapies have been developed ${ }^{1,2}$. Monoclonal antibodies (mAbs) are a promising class of therapeutics against SARS-CoV-2 infection. To date, multiple studies have reported the discovery and characterization of potent neutralizing mAbs, most of which target the receptor-binding domain (RBD) of the spike (S) glycoprotein of SARS-CoV-2 and block the binding between the $\mathrm{S}$ protein and the host receptor, ACE2 (refs. ${ }^{3,4}$ ). There are also some neutralizing $\mathrm{mAbs}$ that target nonblocking epitopes of the RBD or N-terminal domain (NTD) of the S protein 5,6 . However, when selective pressure is applied in immunotherapies, the emergence of escape mutants is a major concern ${ }^{7}$

Thus far, several SARS-CoV-2 variants have become dominant. SARS-CoV-2 B.1, with the D614G mutation in the S protein, has emerged as a dominant variant with increased infectivity but does not appear to be antigenically distinct ${ }^{8,9}$. B.1.1.7 (also known as 501Y.V1 or the Alpha variant) contains eight $\mathrm{S}$ mutations in addition to D614G, including N501Y in the RBD, two deletions in the NTD (69-70del and 144del) and the P681H mutation near the furin cleavage site, which lead to enhanced transmissibility ${ }^{10-13}$. The South African B.1.351 variant (also known as 501Y.V2 or the Beta variant) includes nine mutations (L18F, D80A, D215G, R246I, K417N, E484K, N501Y, D614G and A701V) in the S protein, of which three mutations (K417N, E484K and N501Y) are located in the RBD and increase binding affinity to ACE2 (refs. ${ }^{14-16}$ ). This variant is refractory to neutralization by many mAbs targeting the NTD and
$\mathrm{RBD}$. It is also remarkably more resistant to neutralization by convalescent plasma and vaccinee sera ${ }^{17}$. B.1.525 has key $S$ mutations (A67V, 69-70del, 144del, E484K, D614G, Q677H and F888L) ${ }^{18,19}$. The B.1.617.2 variant (Delta variant) rapidly spreading throughout India carries L452R and E484Q in the RBD. It is reported that the Delta variant is 6.8 -fold more resistant to neutralization by sera from individuals who are COVID-19 convalescent or vaccinated ${ }^{20}$.

To protect against the mutational virus escape, a cocktail strategy (i.e., the administration of two human antibodies that bind to different epitopes of the $\mathrm{S}$ protein) has been reported ${ }^{21-24}$. However, the formulation of such cocktails is complicated, and it increases manufacturing costs and volumes, indicating that it is not an ideal strategy to meet the high demand for COVID-19 therapeutics. Bispecific mAbs (bsAbs) take advantage of the binding of two distinct $\mathrm{mAb}$ and can target two different antigen-binding sites with one molecule. This dual-targeting concept has been successfully evolved alongside the landmark advances in the fields of antibody engineering and antibody biology, leading to the extensive collection of over $100 \mathrm{bsAb}$ formats known today ${ }^{25}$. The bsAb strategy is widely used in the treatment of cancer and inflammatory disor$\operatorname{ders}^{26,27}$, and a limited number of cases have been reported for viral infection diseases ${ }^{28-30}$. Several studies have been proved that bsAbs exhibit enhanced breadth and potency than parental $\mathrm{mAbs}^{31-33}$. Therefore, bsAb is an effective strategy in drug development. There are numerous architectures to develop bsAbs, and different design strategies result in different outcomes ${ }^{25,34}$. Considering the

'CAS Key Laboratory of Pathogen Microbiology and Immunology, Institute of Microbiology, Chinese Academy of Sciences, Beijing, China. ${ }^{2}$ University of Chinese Academy of Sciences, Beijing, China. ${ }^{3}$ College of Veterinary Medicine, China Agricultural University, Beijing, China. ${ }^{4}$ Department of Pathogen Microbiology, School of Basic Medical Sciences, Capital Medical University, Beijing, China. ${ }^{5}$ School of Life Sciences, University of Science and Technology of China, Hefei, Anhui, China. ' ${ }^{2}$ Laboratory of Animal Infectious Diseases, College of Animal Sciences and Veterinary Medicine, Guangxi University, Nanning, China. ${ }^{7}$ Institute of Medical Biology, Chinese Academy of Medical Sciences and Peking Union Medical College, Kunming, Yunnan, China. ${ }^{8} \mathrm{NHC}$ Key Laboratory of Biosafety, National Institute for Viral Disease Control and Prevention, Chinese Center for Disease Control and Prevention, Beijing, China. IInstitute of Biophysics, Chinese Academy of Sciences, Beijing, China. ${ }^{10}$ School of Public Health (Shenzhen), Sun Yat-sen University, Guangzhou, China. "Laboratory of Protein Engineering and Vaccines, Tianjin Institute of Industrial Biotechnology, Chinese Academy of Sciences, Tianjin, China. ${ }^{12}$ Laboratory of Structural Biology, Tianjin Institute of Industrial Biotechnology, Chinese Academy of Sciences, Tianjin, China. ${ }^{13}$ These authors contributed equally: Zhaohui Li, Shihua Li, Gen Zhang, Weiyu Peng.凶e-mail: tansg@im.ac.cn; gaof@im.ac.cn; gaofeng@tib.cas.cn; wuyan@ccmu.edu.cn 
a
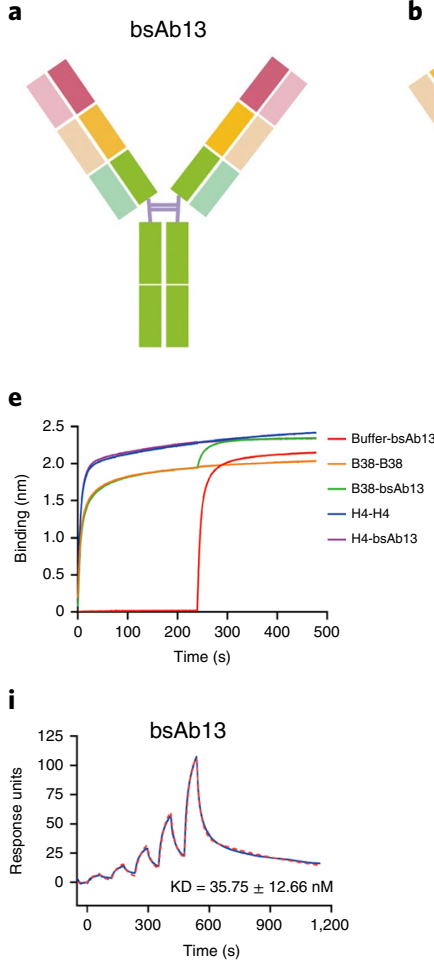

b

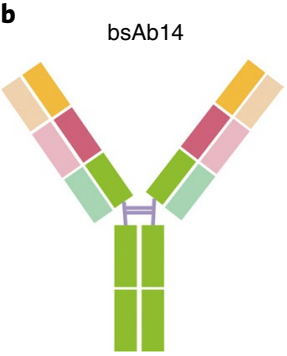

f

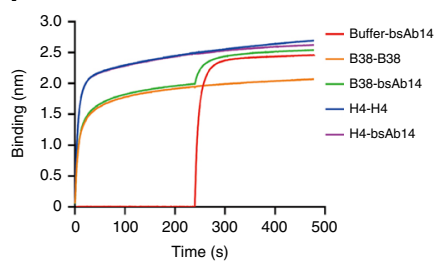

j

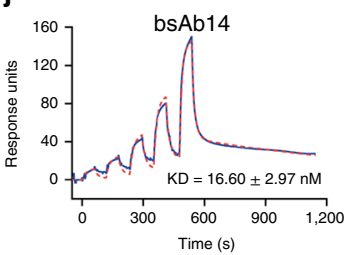

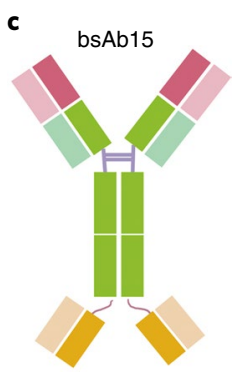

g

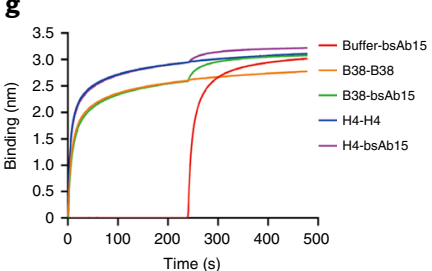

k

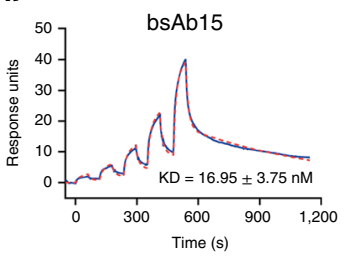

d

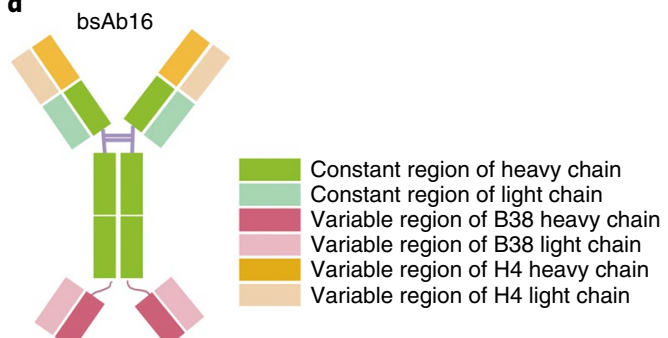

Fig. 1 | Design and characterization of bsAbs. a-d, Formats of the bsAbs designed. The formats of bsAb13 (a) and bsAb14 (b) are DVD-Ig, whereas bsAb15 (c) and bsAb16 (d) are IgG-ScFv. Antibody domains are colored according to their architecture (green, constant domain of the heavy chain; light green, constant domain of the light chain; deep pink, variable heavy chain of B38; light pink, variable light chain of B38; yellow, variable heavy chain of H4; and light yellow, variable light chain of $\mathrm{H} 4$ ). $\mathbf{e - h}$, Competitive binding of parental mAbs and bsAbs. Biotinylated antigen (SARS-CoV-2 RBD) was immobilized on streptavidin sensors and then saturated with B38 or H4, followed by bsAbs. i-I, Binding affinity analysis of bsAbs to SARS-CoV-2 RBD. bsAbs were immobilized on protein A chips and tested for real-time association and dissociation of the RBD. The equilibrium dissociation constant (KD) was calculated using the association constant $(K a)$ and dissociation constant $(K d)$ with a two-state reaction model. The solid blue line is the actual operation curve, and the dashed red line is the fitted curve. Each experiment was repeated twice, and the results shown are means \pm s.d. See also Extended Data Fig. 1.

pharmacokinetic properties or effector functions, the Fc region is normally retained in the bsAb design.

A pair of noncompeting human neutralizing antibodies has been discovered, B38 and $\mathrm{H} 4$, which protect mice from SARS-CoV-2 challenge and have the potential ability to avoid immune escape ${ }^{3}$. In this study, four bsAbs were designed based on these two parental $m A b s$. Two immunoglobulin $\mathrm{G}$ (IgG) single-chain variable fragment ( $\mathrm{ScFv}$ )-format bsAbs (bsAb15 and bsAb16) exhibited more potent neutralizing efficacy against both pseudo and authentic SARS-CoV-2 viruses compared to the parental mAbs or any of other designed bsAbs. Furthermore, in vivo experiments indicated that bsAb15 effectively protects both mice and rhesus macaques from SARS-CoV-2 infection. In addition, viral escape mutants were screened in the presence of antibodies. BsAb15 led to less escape mutations compared to single-antibody treatment or antibody cocktail therapy. Using bsAb15, we also identified its binding affinity and neutralizing ability toward escape mutants. BsAb15 could bind to the RBD with escape mutations apart from the Beta variant (K417N and $\mathrm{E} 484 \mathrm{~K}$ mutations in the RBD). In line with the binding assays, bsAb15 displayed higher efficacy ( $\mathrm{IC}_{50}$ numbers are lower) toward mutant pseudoviruses than parental mAbs, except for the Beta variant.

\section{Results}

Design and characterization of bsAbs. To obtain functional bsAb, we engineered two symmetric formats based on two previously identified human mAbs, B38 and $\mathrm{H} 4\left(\right.$ ref. $^{3}$ ). One format is dual-variable domain immunoglobulin (DVD-Ig), whereas the other is IgG-ScFv.
For each type of bsAb, we designed two constructs with the position of the variable region of the mAbs exchanged with one another (Fig. 1a-d). All of these bsAbs folded correctly, and soluble proteins could be obtained (Extended Data Fig. 1). To test whether the two variable regions on these bsAb interfered with each other and were still available to access RBD antigens, we performed a competition assay by biolayer interferometry. The RBD protein was immobilized on SA sensors and saturated with B38 or H4. Then, bsAb was added to the sensor. We found that bsAb15 and bsAb16 could still bind to the RBD after saturation with the first mAb, suggesting that both RBD binding sites on these bsAbs were exposed (Fig. 1g,h). In contrast, the $\mathrm{H} 4$ binding epitope was embedded in bsAb13 and bsAb14, and no binding could be detected after saturation with the $\mathrm{H} 4 \mathrm{mAb}$. The B38 epitope in these two bsAbs was still exposed, and additional binding to the RBD could be observed. Further, the $\mathrm{H} 4$ epitope was also blocked in bsAb13 and bsAb14 when the RBD proteins were saturated with B38 (Fig. 1e,f). Additionally, an avidity assay using surface plasmon resonance (SPR) confirmed that these bsAbs display similar affinity to the RBD compared to B38 or H4 (Fig. 1i-1).

bsAbs neutralize virus. To identify the neutralization properties of the four bsAbs, we performed neutralization assays with both pseudovirus and authentic virus. Among the bsAbs, bsAb15 and bsAb16 exhibited comparable neutralization potencies against SARS-CoV-2 pseudovirus, with half-maximum inhibitory concentration $\left(\mathrm{IC}_{50}\right)$ values of 0.18 and $0.24 \mathrm{nM}$, respectively, which displayed substantially lower values than that of cocktail or parental mAbs. bsAb13 
a

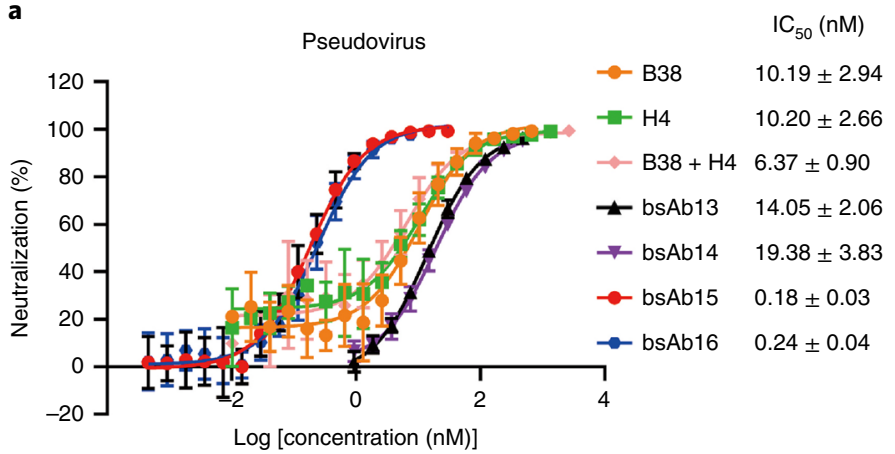

b

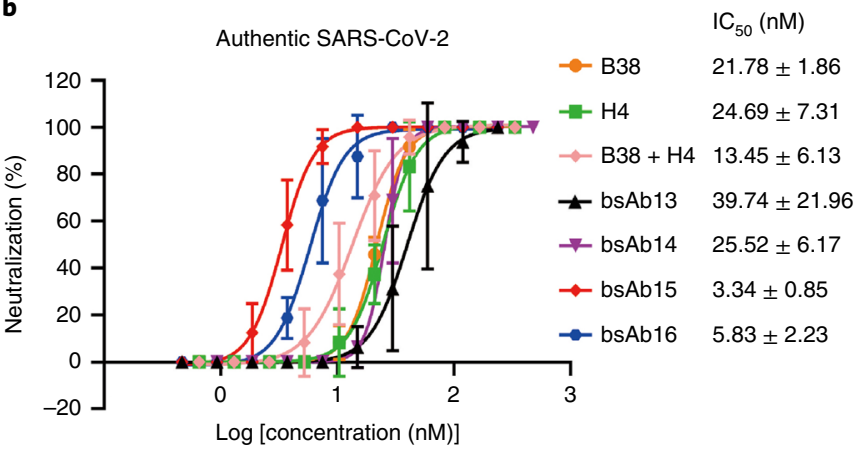

Fig. 2 | Neutralization of antibodies against pseudovirus and authentic virus. a,b, Two parental mAbs and four bsAbs were analyzed using the vesicular stomatitis virus-based pseudovirus system $(n=3)(\mathbf{a})$ and authentic SARS-CoV-2 $(n=2)(\mathbf{b})$. The IC 50 was determined by log (inhibitor) response of nonlinear regression and is displayed as the mean \pm s.d.

and bsAb14 displayed similar neutralizing ability to $\mathrm{B} 38$ or $\mathrm{H} 4$ against pseudovirus, with $\mathrm{IC}_{50}$ values of 14.05 and $19.38 \mathrm{nM}$, respectively (Fig. 2a). In the authentic SARS-CoV-2 infection system, the bsAbs exhibited similar neutralization trends (Fig. 2b). Specifically, bsAb15 and bsAb16 neutralized the authentic SARS-CoV-2 with $\mathrm{IC}_{50}$ values of 3.34 and $5.83 \mathrm{nM}$, respectively. In contrast, bsAb13 showed relatively weak neutralization to authentic SARS-CoV-2 compared to $\mathrm{B} 38$ or $\mathrm{H} 4$ alone, with an $\mathrm{IC}_{50}$ of $39.74 \mathrm{nM}$. BsAb14 displayed similar neutralizing ability to $\mathrm{B} 38$ or $\mathrm{H} 4$, with an $\mathrm{IC}_{50}$ of $25.52 \mathrm{nM}$.

Antibody-dependent enhancement (ADE) is one of the concerns in antibody treatment. Therefore, we evaluated the antibody-dependent viral entry (ADE of viral entry) of bsAb15 using the CD32A H131-CHOK1 cell system in vitro as an indicator of $\mathrm{ADE}$. However, no ADE of viral entry was observed compared to the positive control antibody (Extended Data Fig. 2). Because bsAb15 exhibited the most potency in both pseudo- and authentic SARS-CoV-2 infections and did not trigger ADE of viral entry, we next evaluated in vivo protection efficacy in animal models.

bsAb15 protects against SARS-CoV-2 infection in vivo. To assess the protection efficacy of bsAb15, we performed both prophylactic and protective experiments in a replication-deficient adenovirus (Ad5-hACE2) mouse model (Fig. 3a). In the pre-exposure group, mice were intraperitoneally injected with $50 \mathrm{mg} \mathrm{kg}^{-1}$ bsAb15 and then challenged $24 \mathrm{~h}$ later with $2 \times 10^{6}$ median tissue culture infectious dose $\left(\mathrm{TCID}_{50}\right)$ of SARS-CoV-2. In the post-exposure group, mice were treated $12 \mathrm{~h}$ after infection with SARS-CoV-2 with $50 \mathrm{mg} \mathrm{kg}^{-1}$ bsAb15. All of the mice were sacrificed at 3 dpi (days postinfection), and the viral loads in the lungs of each group were quantified using RT-qPCR. Both the prophylactic group and protective group displayed significantly reduced viral titers in the lung compared to the PBS control group (Fig. 3b). To compare the efficiency of bsAb15, cocktail and parental mAbs, $25 \mathrm{mg} \mathrm{kg} \mathrm{kg}^{-1}$ antibodies (B38, H4, combination of B38 and $\mathrm{H} 4$ or bsAb15) were administrated to each group of mice $12 \mathrm{~h}$ after challenge with authentic SARS-CoV-2. We clearly observed that the viral RNA levels in the bsAb15 treatment group were significantly lower than the cocktail and parental mAb treatment groups (Fig. 3c).

Pathology analyses of the lungs of the infected animals were also performed (Fig. 3d). Histopathological observations indicated that the lung tissues from the placebo group mice at $3 \mathrm{dpi}$ displayed moderate interstitial pneumonia. Lung lesions showed alveolar septa thickening, inflammatory cell accumulation (including lymphocytes, macrophages, and neutrophils) and homogeneously pink infiltration in partial alveolar cavities. Meanwhile, multifocal hemorrhage was obvious in the partial alveolar cavities or bronchiolar cavities. In contrast, no substantial pathological changes were observed in the prophylaxis group. Mice in the treatment group exhibited mild interstitial pneumonia compared to the control group. The area percentages of pulmonary inflammation in the lungs of the PBS control group, prophylaxis group and treatment group were estimated by pathological quantitative analysis software as $40.67 \%, 12.61 \%$ and $26.54 \%$, respectively, displaying the same trends as the histopathological observations (Extended Data Fig. 3).

We further evaluated protection efficacy in a rhesus macaque SARS-CoV-2 infection model in both prophylaxis and treatment settings (Fig. 4a). In the prophylaxis group, $50 \mathrm{mg} \mathrm{kg}^{-1}$ bsAb15 was administered to four animals $12 \mathrm{~h}$ before challenge with $100 \mu \mathrm{l} 5.0$ $\operatorname{lgCCID}_{50}$ SARS-CoV-2 virus. In the treatment group, bsAb15 was administered twice at $12 \mathrm{~h}$ and $84 \mathrm{~h} \mathrm{dpi}$, respectively. High doses $\left(50 \mathrm{mg} \mathrm{kg}^{-1}\right)$ and low doses $\left(25 \mathrm{mg} \mathrm{kg}^{-1}\right)$ of bsAb15 were tested. Throat swabs and nasal swabs were collected daily. The peak of viral loads of both throat swabs and nasal swabs in the placebo group appeared at $4 \mathrm{dpi}$, including both genomic and subgenomic RNA

Fig. 3 Therapeutic and prophylactic efficacy of bsAb15 in a mouse model of SARS-CoV-2 infection. a, Immunization strategies of bsAb15 in Ad5-hACE2 virus-infected BALB/c mice. Prophylaxis and treatment of bsAb15 in the mouse model were performed in study A $(n=5)$ and study B $(n=5)$. b, Impact of prophylaxis and treatment with $50 \mathrm{mg} \mathrm{kg}^{-1}$ bsAb15 on RNA levels in mouse lungs detected by primers of the ORF1ab gene and $\mathrm{N}$ gene, respectively. Data are shown as means with s.d. and were analyzed by one-way analysis of variance ( $\left.{ }^{\star \star \star} P<0.0001\right)$. c, Comparison of the impact of treatment with $25 \mathrm{mg} \mathrm{kg}{ }^{-1}$ $\mathrm{B} 38, \mathrm{H} 4$, cocktail or bs Ab15 on the RNA levels in mice lungs $(n=6)$ detected by primers for the $\mathrm{N}$ gene and ORF1ab gene, respectively. Data are shown as the means with s.d. and were analyzed by one-way analysis of variance (NS, $P>0.05$; $\left.{ }^{\star \star \star} P=0.0001 ; * \star \star \star P<0.0001\right)$. d, Representative histopathology of the lung tissues from pre-exposure, postexposure and placebo mice, which were killed and necropsied on 3 dpi. Samples were stored in formalin for 7 days and then embedded in paraffin. Sectioned samples were stained with hematoxylin and eosin (H\&E). In the placebo group, lung lesions showed alveolar septa thickening (black arrows), inflammatory cell accumulation (including lymphocytes, macrophages and neutrophils (blue arrows)) and homogeneously pink infiltration in partial alveolar cavities (green arrows). Meanwhile, multifocal hemorrhage was seen in the partial alveolar cavities (yellow arrow) or bronchiolar cavities (red arrow). The pathological sections in the treatment group displayed infiltrating macrophages, lymphocytes and neutrophils (blue arrows) accompanied by alveolar septa and thick alveolar walls (black arrows). Scale bars are labeled on each panel. See also Extended Data Fig. 3. 
(sgRNA) samples (Fig. 4b). In contrast, bsAb15-treated animals displayed lower viral loads in both throat and nasal swabs. For all animals receiving bsAb15 prophylaxis and treatment, sgRNA in throat swabs and nasal swabs was barely detected from $1 \mathrm{dpi}$ and $3 \mathrm{dpi}$, respectively. Both treatment groups displayed similar kinetics of virus clearance except for one monkey (20457) in the high-dose group, indicating that $25 \mathrm{mg} \mathrm{kg}^{-1}$ and $50 \mathrm{mg} \mathrm{kg}^{-1} \mathrm{mAbs}$ presented similar efficacy in this study.

To further evaluate the pharmacokinetics of bsAb15, one dose of the antibody (7.5-75 mg kg-1) was intravenously administered to Macaca fascicularis. No abnormal clinical manifestations were observed in any group. The pattern of bsAb15 concentrations in

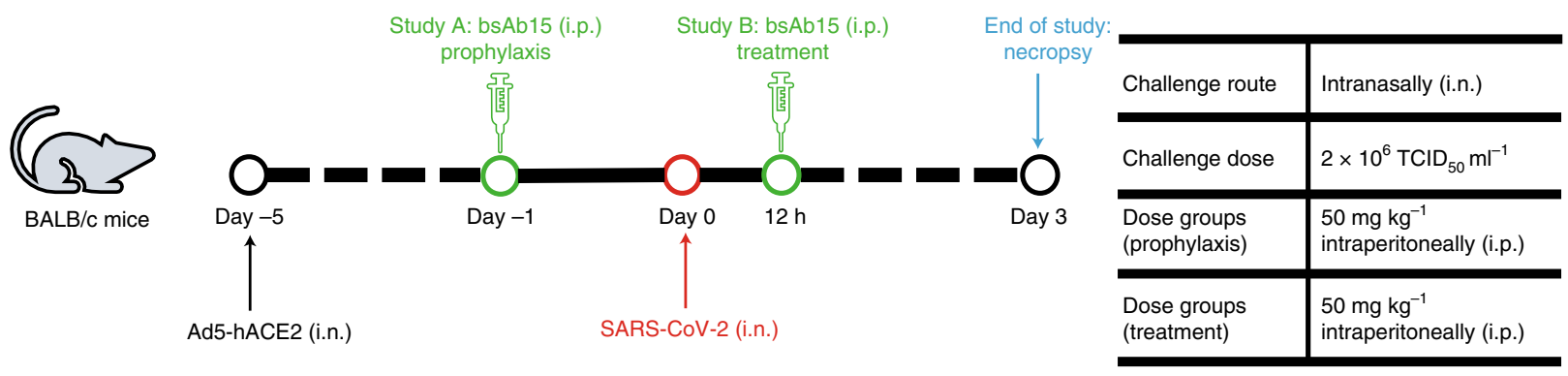

b

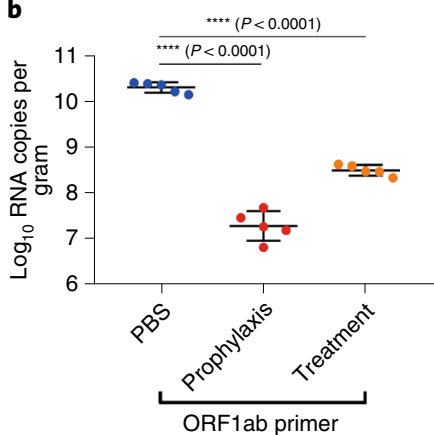

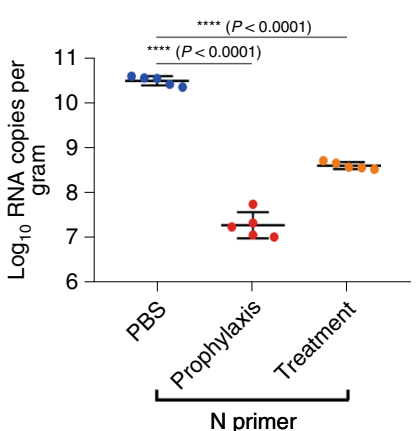

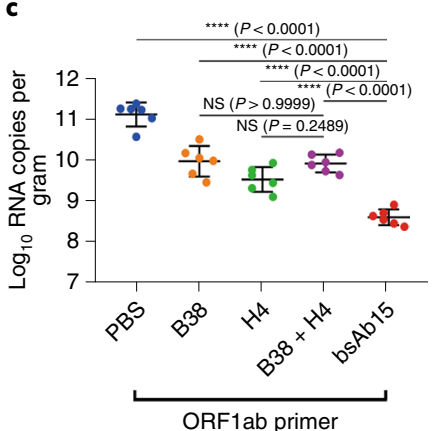

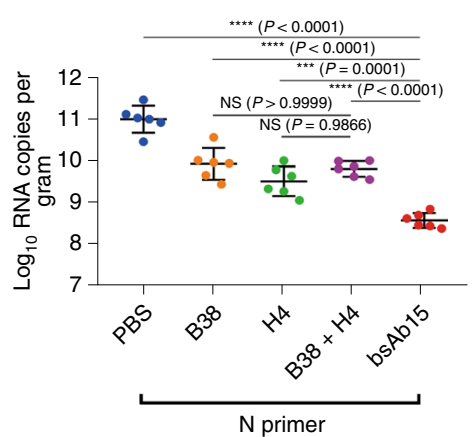

d

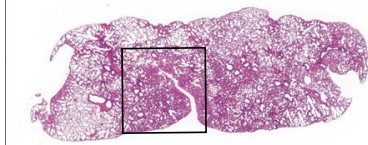

Prophylaxis group
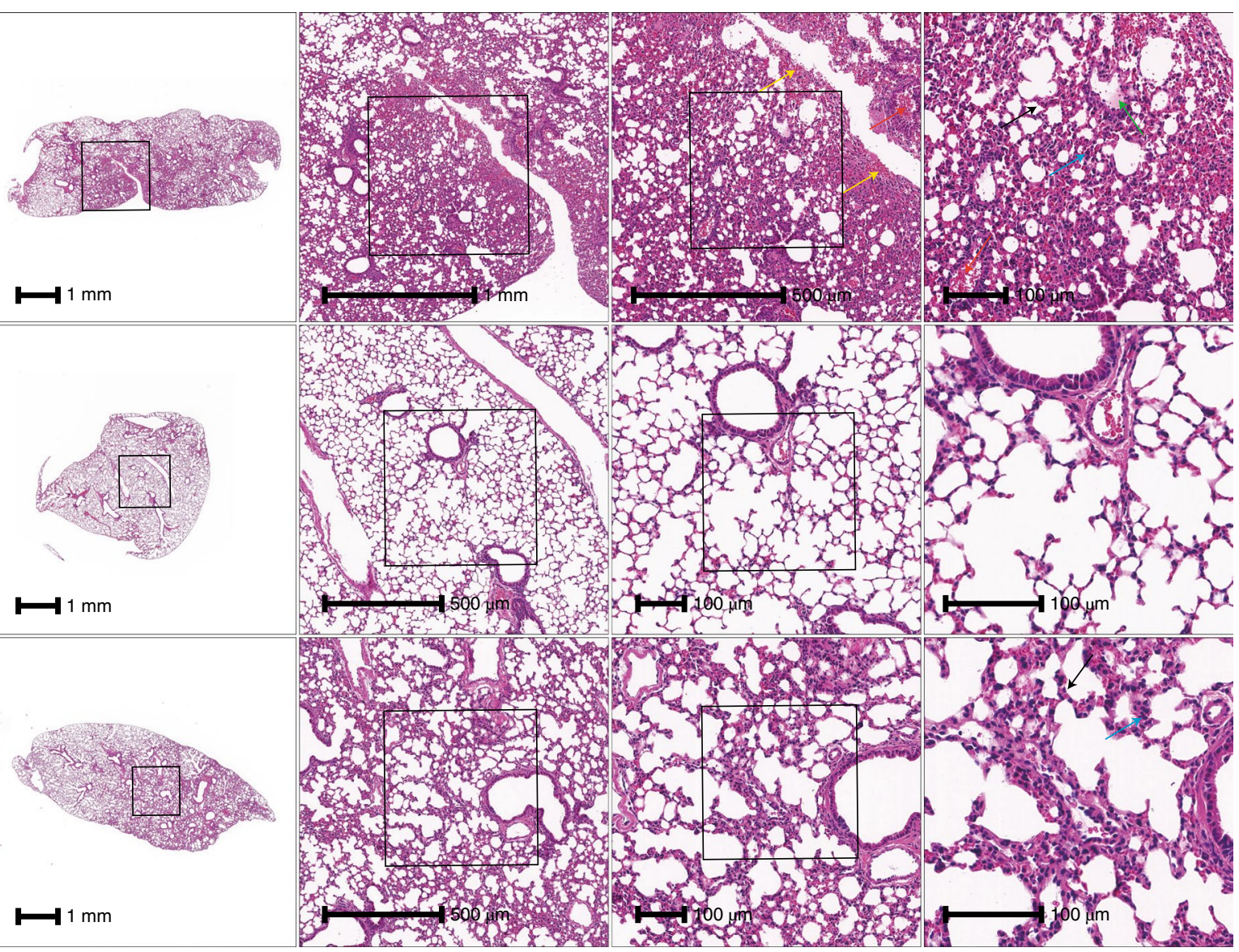

Treatment group

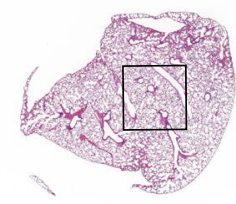

$\boldsymbol{|} 1 \mathrm{~mm}$

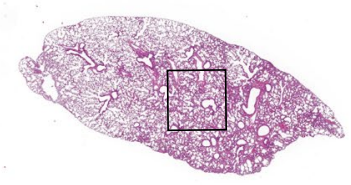

$\longrightarrow 1 \mathrm{~mm}$ 
a

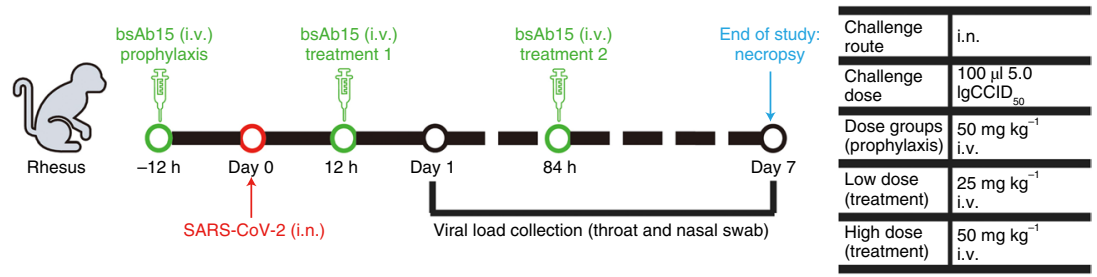

b

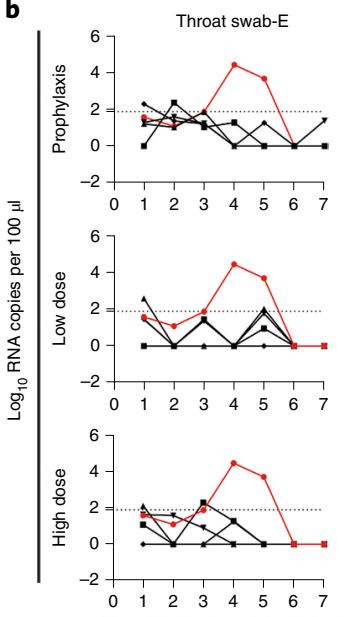

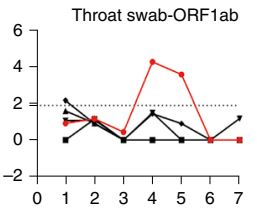
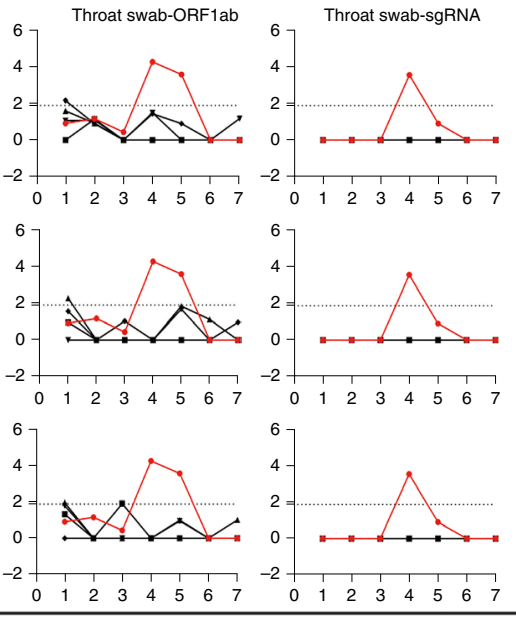
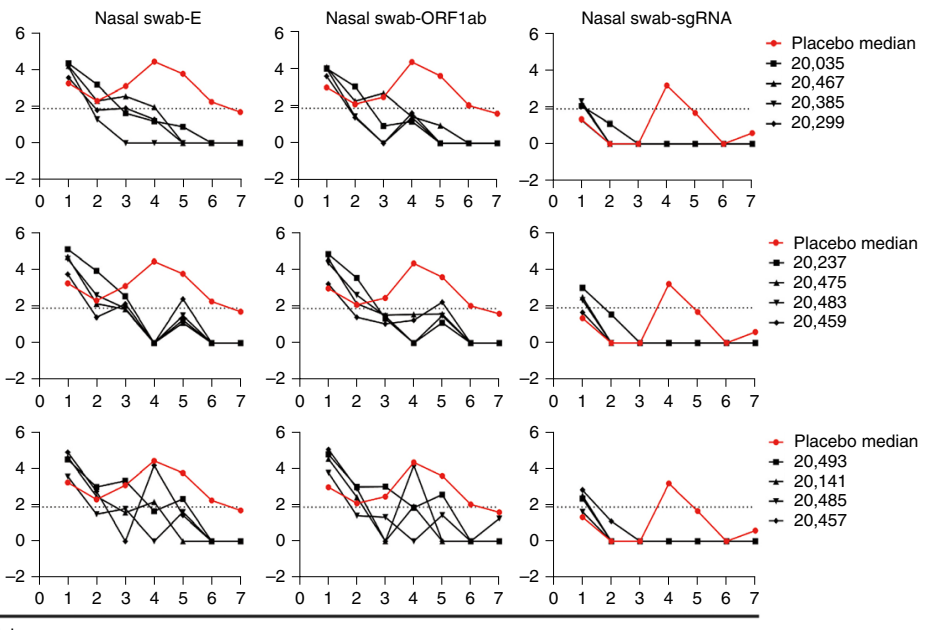

Fig. 4 | Protection efficacy of bsAb15 in rhesus macaques. a, Immunization strategy of bsAb15 in the rhesus macaque for both prophylaxis and treatment. b. Impact of bsAb15 prophylaxis and treatment on viral genomic RNA (E and ORF1ab) and sgRNA in throat swabs and nasal swabs. The dashed line indicates the threshold of detection. i.n., intranasal; i.v., intravenous. See also Extended Data Fig. 4.

the serum among male and female animals was similar, with linear pharmacokinetic characteristics (Extended Data Fig. 4).

Escape mutant screening. The rational design of bsAb is to avoid escape mutants. Thus, we next selected escape mutants under pressure of single antibodies, antibody combinations and bsAb using SARS-CoV-2 virus (Fig. 5a). Because these mAbs target the RBD, we focused on mutations that occur in the RBD region. In the presence of $\mathrm{H} 4$, the escape mutant E484A became readily fixed in the population by the third passage, representing $91.87 \%$ of sequencing reads, and was resistant to $\mathrm{H} 4$ doses of up to $500 \mu \mathrm{g} \mathrm{ml}^{-1}$. For B38, the escape mutants N460S and V407A were dominant after the third passage, representing $88.89 \%$ and $18.18 \%$ of sequencing reads, respectively. Under the pressure of a B38 and H4 cocktail, the highest antibody concentration of cytopathic effect (CPE) was the same after three passages, and $97.37 \%$ of N460S and $8.7 \%$ of $\mathrm{Q} 474 \mathrm{H}$ mutant viruses were observed by passage 3. The pseudovirus neutralization assay suggested that $\mathrm{Q} 474 \mathrm{H}$ did not decrease the neutralizing ability of mAbs (Extended Data Fig. 5). In contrast, the bsAb15 group displayed similar qualitative percentages of CPE within both passage 1 and passage 3 (Fig. 5b), and no dominant escape mutants were observed in the RBD region (Fig. $5 \mathrm{c}$ ), indicating that the bsAb may lead to less selective pressure compared to single-antibody treatment or antibody cocktail therapy. There were also several nonsynonymous changes in the $\mathrm{S}$ protein outside the RBD region (Supplementary Table 1).

Biochemical and in vitro neutralizing properties of bsAb15. To verify which mutants may result in the loss of activity of the antibodies, we expressed the RBD proteins with substitution mutations and measured the affinity between the RBD mutants and antibodies by SPR (Fig. 6a). The analysis revealed that $\mathrm{K} 417 \mathrm{~N}$ resulted in loss of B38 binding, N460S decreased the B38 binding and E484K and
E484A abolished H4 binding. In contrast, bsAb15 maintained avidity to these RBD mutants. The N501Y mutation did not affect the interaction between the RBD and antibodies. Notably, this mutation substantially improved the binding of $\mathrm{B} 38$ and bsAb15 compared to the wild-type (WT) RBD. However, bsAb15 lost binding to the RBD from the Beta variant, which contains K417N, E484K and N501Y mutations. Among these substitutions, K417N and E484K are likely the key residues resulting in loss of avidity (Fig. 6a).

We further evaluated the neutralizing ability of bsAb15 in vitro by using SARS-CoV-2 pseudoviruses. In line with the binding assays, apart from the Beta variant, bsAb15 retained efficacy against pseudoviruses bearing escape mutations that made them resistant to the individual mAbs (Fig. $6 \mathrm{~b}$ and Table 1). Specifically, bsAb15 inhibited the pseudovirus carrying the $\mathrm{K} 417 \mathrm{~N}$ mutation at nanomolar concentrations $\left(\mathrm{IC}_{50}=9.875 \mathrm{nM}\right)$, whereas $\mathrm{B} 38$ lost its neutralizing ability. For the N460S mutation selected under pressure, bsAb15 exhibited a 490 -fold better $\mathrm{IC}_{50}$ than B38. Moreover, bsAb15 maintained potent neutralizing efficacy to pseudovirus with a mutation at residue 484 (E484K, E484A, the Kappa and Eta variants, respectively). In contrast, these pseudoviruses abolished the neutralizing ability of H4. Notably, bsAb15 exhibited potent neutralizing efficacy to Delta pseudovirus, with an $\mathrm{IC}_{50}$ value lower than $0.015 \mathrm{nM}$. Structural analysis supported the neutralizing and binding results (Fig. 6c). Specifically, K417 and N460 are located on the epitope of B38, whereas E484 is at the edge of the B38 epitope, and N501 is beyond the B38 epitope.

\section{Discussion}

Several monoclonal antibodies targeting the SARS-CoV-2 $\mathrm{S}$ protein have been developed and used in clinics or clinical trials against COVID-19. New strategies against viral evasion and that reduce the risk of escape mutations should be developed, because escape mutations threatening the efficacy of single mAbs have already 


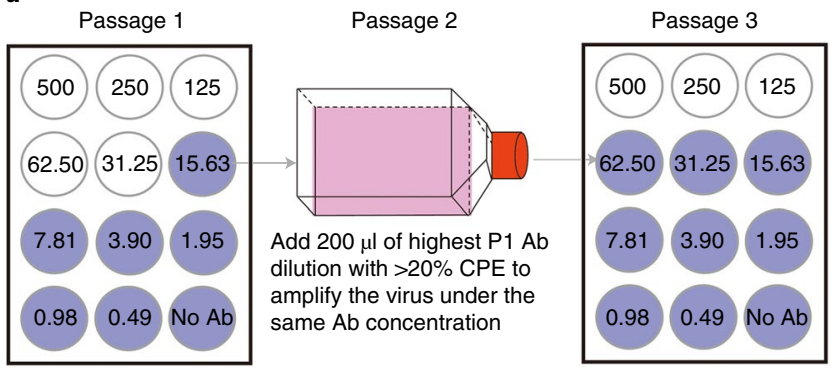

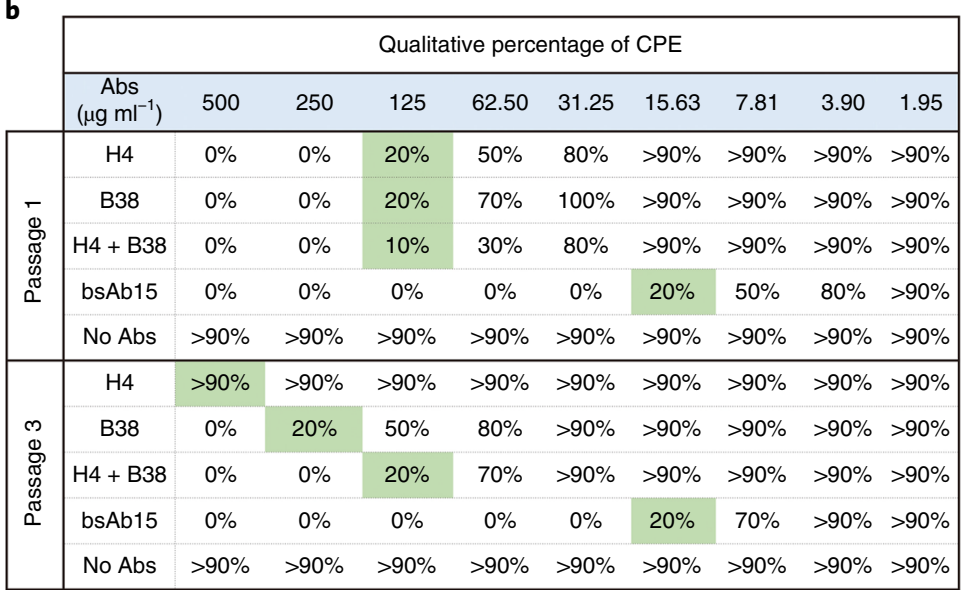

c

\begin{tabular}{|c|c|c|c|c|}
\hline Position in S gene & 1,220 & 1,379 & 1,422 & 1,451 \\
\hline Reference nucleotide & $\mathrm{T}$ & A & G & A \\
\hline Variant nucleotide & C & G & $\mathrm{T}$ & C \\
\hline Position in S protein & 407 & 460 & 474 & 484 \\
\hline Reference residue & V & $\mathrm{N}$ & Q & $E$ \\
\hline Variant residue & A & $S$ & $\mathrm{H}$ & A \\
\hline $\mathrm{H} 4125 \mu \mathrm{g} \mathrm{ml}^{-1}$ & $<1 \%$ & $<1 \%$ & $<1 \%$ & $<1 \%$ \\
\hline B38 $125 \mu \mathrm{g} \mathrm{ml}^{-1}$ & $<1 \%$ & $<2 \%$ & $<1 \%$ & $<1 \%$ \\
\hline $\mathrm{H} 4+\mathrm{B} 38125 \mu \mathrm{g} \mathrm{ml}^{-1}$ & $<1 \%$ & $0 \%$ & $<1 \%$ & $0 \%$ \\
\hline bsAb15 $15.63 \mu \mathrm{g} \mathrm{ml}^{-1}$ & $<1 \%$ & $<1 \%$ & $<1 \%$ & $0 \%$ \\
\hline No Abs (virus only) & $<1 \%$ & $<1 \%$ & $<1 \%$ & $<1 \%$ \\
\hline $\mathrm{H} 4500 \mu \mathrm{g} \mathrm{ml}^{-1}$ & $<1 \%$ & $<1 \%$ & $<1 \%$ & $91.32 \%$ \\
\hline B38 $250 \mu \mathrm{g} \mathrm{ml}^{-1}$ & $18.18 \%$ & $88.89 \%$ & $<1 \%$ & $0 \%$ \\
\hline $\mathrm{H} 4+\mathrm{B} 38125 \mu \mathrm{g} \mathrm{ml}^{-1}$ & $0 \%$ & $97.37 \%$ & $8.70 \%$ & $0 \%$ \\
\hline bsAb15 $15.63 \mu \mathrm{g} \mathrm{ml}^{-1}$ & $<1 \%$ & $0 \%$ & $<1 \%$ & $0 \%$ \\
\hline No Abs (virus only) & $<1 \%$ & $<1 \%$ & $<1 \%$ & $<1 \%$ \\
\hline
\end{tabular}

Fig. 5 | Escape mutant screening under the pressure of antibodies. a, Schematic of the escape mutant screening process. Authentic SARS-CoV-2 was passaged in the presence of antibodies (B38, H4, B38 + H4 or bsAb15) with serial dilutions on Vero E6 cells. CPE was used to monitor virus replication. b, Quantitative percentage of CPE under different concentrations of antibodies during passage 1 and passage 3. Less CPE was observed in the presence of bsAb15 compared to cells containing B38, H4, or B38 and $\mathrm{H} 4$ cocktail. Green boxes indicate dilutions that were passaged and sequenced in passage 1 or sequenced in passage 3. c, Next-generation sequencing of virus RNA from passage 1 and passage 3. Variant nucleotides and corresponding residues are shown, and the qualitative percentage of CPE observed in each dilution is presented (dark purple, $\geq 20 \%$ CPE; light pink, <20\% CPE). A group without antibody was used as the negative control. Ab, antibody; P1, passage 1.

been detected in viruses circulating in humans and minks $s^{35,36}$. Construction of distinct mAbs into one molecule is a promising therapeutic strategy. On one hand, bsAbs present potent neutralizing efficacy and protection against SARS-CoV-2 infection. On the other hand, viruses can escape much more frequently under pressure by a single antibody rather than by a bsAb in vitro. Moreover, bsAbs are a more cost-effective tactic than using single or cocktail $\mathrm{mAbs}$ in drug development, which can fulfil the augmented requirements of production capacity due to COVID-19-related vaccines and therapeutics.

The bsAbs usually consist of two antibodies targeting two antigens or two different epitopes. The rationale for $a \mathrm{bsAb}$ is that it is unlikely that one mutation in the $S$ protein will simultaneously render ineffectiveness of both antibodies. However, mutations that simultaneously emerge within both epitopes may abolish the neutralizing ability of bsAbs. In this study, it is clear that the K417N mutation resulted in the loss of binding by B38, and variants of E484 abolished $\mathrm{H} 4$ binding. The combination of mutations at these two sites (B.1.351 or Beta variant) resulted in bsAb15 resistance. We speculate that bsAb15 may display little neutralization to P.1 (501Y. $\mathrm{V} 3$, or Gamma variant), because it also carries mutations at these two residues (K417T and E484K) ${ }^{37}$.

One of the advantages of bsAb is preventing the escape mutants. In this study, escape mutations occur under the selective pressures of parental mAbs or the antibody cocktail. In contrast, no mutations appear in the RBD region in the presence of bsAb15 (at least after three passages). A similar phenomenon has been reported for in vitro escape mutation selection assays in the presence of Food and Drug Administration-approved mAbs. For the cocktail antibodies REGN-COV2, serial passages of pseudovirus encoding the SARS-CoV-2 S protein in the presence of drugs identified several escape variants to casirivimab (K417E, Y453F, L455F, F486V and Q493K) or imdevimab (K444Q and V445A) 22 . Each viral variant exhibiting reduced susceptibility to one $\mathrm{mAb}$ retains susceptibility to the other $\mathrm{mAb}$. In a separate neutralization assay, pseudoviruses with 37 SARS-CoV-2 RBD variants identified as the most common variants in circulation (as of late March 2021) were used to evaluate the neutralizing ability of casirivimab and imdevimab. Casirivimab reduced susceptibility to G476S and S494P variants ${ }^{38}$, and imdevimab reduced susceptibility to the $\mathrm{N} 439 \mathrm{~K}$ variant. However, the combination of mutants at the 417 and 439 residues abrogated the effectiveness of the REGN-COV2 cocktail ${ }^{36}$. For bamlanivimab (developed by Lily), serial passages of SARS-CoV-2 under selective pressure result in four viral variants (E484K, F490S, Q493R and S494P in the RBD), which increase resistance to this drug $^{39}$. Three variants (E484K, F490S and S494P) were observed in a clinical trial. Etesevimab, which contains a similar epitope to $\mathrm{B} 38$, may be resistant to mutations at residue K417. Therefore, it is possible that combination therapies may be challenged by simultaneous mutations of the two epitopes. Moreover, it is notable that $\mathrm{K} 417$ and E484 are two important mutation sites that poses a threat to the efficacy of most neutralizing antibodies developed so far. Therefore, it is essential to identify broad neutralizing parental $\mathrm{mAbs}$ for effective bsAbs development. However, the good news is that our bsAb15 is highly effective to the currently emerging Delta variant. 

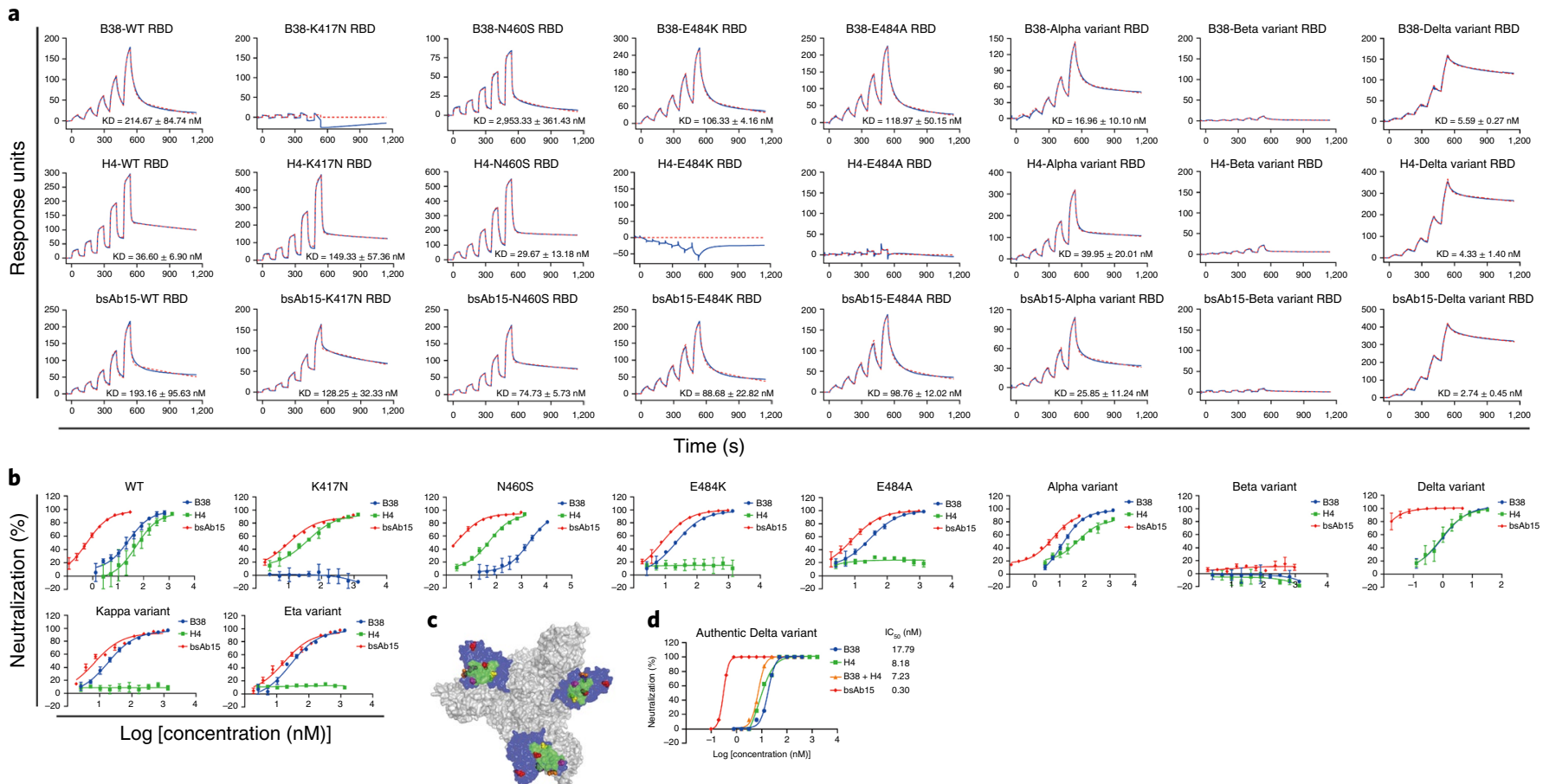

Log [concentration $(\mathrm{nM})]$

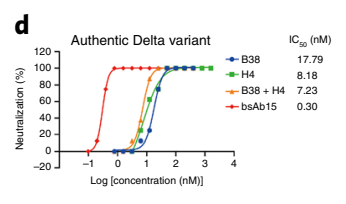

Fig. 6 Affinity characterization and neutralizing ability of bsAb15 against mutant pseudoviruses. a, SPR kinetics analysis of the binding of antibodies to SARS-CoV-2 RBD mutants. Antibody was immobilized on protein A chips and then exposed to diluted SARS-CoV-2 RBD WT or mutants (K417N, N460S, E484K, E484A, N501Y or K417N/E484K/N501Y). The KD values are the mean \pm s.d. in triplicate. b, In vitro neutralization activity of antibodies against WT and mutant pseudoviruses in Vero E6 cells. The curves were fit by the log (inhibitor) response of nonlinear regression and displayed as the means \pm s.d. $(n=3)$. c. The RBD residues mutated in the different variants are indicated as red (K417 and N501), yellow (N460), orange (E484) and magenta (L452 and T478) spheres on the S trimer structure. The RBD (blue) and epitope of B38 (green) are shown in surface representation. d, Neutralization activity of antibodies against authentic Delta virus. The curves were fit by the log (inhibitor) response of nonlinear regression and are displayed as the means \pm s.d. $(n=1)$.

\begin{tabular}{|c|c|c|c|}
\hline $\mathrm{IC}_{50}(\mathrm{nM})$ & B38 & $\mathrm{H} 4$ & bsAb15 \\
\hline WT & $25.67 \pm 8.76$ & $51.19 \pm 12.03$ & $0.61 \pm 0.07$ \\
\hline K417N & NE & $38.18 \pm 9.34$ & $9.88 \pm 3.36$ \\
\hline N460S & $1932 \pm 361.90$ & $51.42 \pm 1.67$ & $3.94 \pm 1.23$ \\
\hline E484K & $21.70 \pm 6.67$ & NE & $7.88 \pm 1.34$ \\
\hline E484A & $28.61 \pm 4.46$ & NE & $8.17 \pm 1.88$ \\
\hline Alpha variant & $12.43 \pm 2.74$ & $55.23 \pm 19.04$ & $5.20 \pm 1.08$ \\
\hline Beta variant & NE & NE & NE \\
\hline Kappa variant & $18.11 \pm 1.27$ & NE & $6.90 \pm 2.25$ \\
\hline Eta variant & $27.22 \pm 8.66$ & NE & $16.58 \pm 3.52$ \\
\hline Delta variant & $0.99 \pm 0.16$ & $0.83 \pm 0.36$ & $<0.015 \pm 0.005$ \\
\hline
\end{tabular}

$\mathrm{ADE}$ is one of the potential hurdles for antibody-based vaccines and therapeutics, which increases the risk of exacerbating viral infection diseases. ADE has been observed in other viruses, including dengue virus ${ }^{40,41}$, feline infectious peritonitis virus ${ }^{42}$, SARS-CoV ${ }^{43}$ and Middle East respiratory syndrome coronavirus ${ }^{44}$. The results from this report show that there is no ADE of viral entry in our bsAb15. We hypothesize that the ScFv linked to the C terminus of bsAb15 Fc may interfere with binding between $\mathrm{Fc}$ and $\mathrm{F} c \gamma \mathrm{R}$, thereby decreasing the possibility of $\mathrm{ADE}$, but the underlying mechanism needs to be further investigated.

\section{Online content}

Any methods, additional references, Nature Research reporting summaries, source data, extended data, supplementary information, acknowledgements, peer review information; details of author contributions and competing interests; and statements of data and code availability are available at https://doi.org/10.1038/ s41590-022-01138-w.

Received: 5 September 2021; Accepted: 13 January 2022; Published online: 28 February 2022

\section{References}

1. Dai, L. et al. A universal design of betacoronavirus vaccines against COVID-19, MERS, and SARS. Cell 182, 722-733 (2020).

2. Shi, R. et al. A human neutralizing antibody targets the receptor-binding site of SARS-CoV-2. Nature 584, 120-124 (2020).

3. $\mathrm{Wu}, \mathrm{Y}$. et al. A noncompeting pair of human neutralizing antibodies block COVID-19 virus binding to its receptor ACE2. Science 368, 1274-1278 (2020).

4. Zost, S. J. et al. Potently neutralizing and protective human antibodies against SARS-CoV-2. Nature 584, 443-449 (2020).

5. Chi, X. et al. A neutralizing human antibody binds to the N-terminal domain of the spike protein of SARS-CoV-2. Science 369, 650-655 (2020).

6. Liu, L. et al. Potent neutralizing antibodies against multiple epitopes on SARS-CoV-2 spike. Nature 584, 450-456 (2020).

7. Kemp, S. A. et al. SARS-CoV-2 evolution during treatment of chronic infection. Nature 592, 277-282 (2021).

8. Hou, Y. J. et al. SARS-CoV-2 D614G variant exhibits efficient replication ex vivo and transmission in vivo. Science 370, 1464-1468 (2020).

9. Korber, B. et al. Tracking changes in SARS-CoV-2 spike: evidence that D614G increases infectivity of the COVID-19 virus. Cell 182, 812-827 (2020).

10. Hoffmann, M., Kleine-Weber, H. \& Pohlmann, S. A multibasic cleavage site in the spike protein of SARS-CoV-2 is essential for infection of human lung cells. Mol. Cell 78, 779-784 (2020). 
11. Starr, T. N. et al. Deep mutational scanning of SARS-CoV-2 receptor binding domain reveals constraints on folding and ACE2 binding. Cell 182, 1295-1310 (2020).

12. Brown, K. A. et al. S-Gene target failure as a marker of variant B.1.1.7 among SARS-CoV-2 isolates in the greater Toronto area, December 2020 to March 2021. JAMA 325, 2115-2116 (2021).

13. Peacock, T. P. et al. The furin cleavage site in the SARS-CoV-2 spike protein is required for transmission in ferrets. Nat. Microbiol. 6, 899-909 (2021).

14. Tegally, H. et al. Detection of a SARS-CoV-2 variant of concern in South Africa. Nature 592, 438-443 (2021).

15. Hoffmann, M. et al. SARS-CoV-2 variants B.1.351 and P.1 escape from neutralizing antibodies. Cell 184, 2384-2393 (2021).

16. Zhou, D. et al. Evidence of escape of SARS-CoV-2 variant B.1.351 from natural and vaccine-induced sera. Cell 184, 2348-2361 (2021).

17. Wang, P. et al. Antibody resistance of SARS-CoV-2 variants B.1.351 and B.1.1.7. Nature 593, 130-135 (2021).

18. Ozer, E. A. et al. High prevalence of SARS-CoV-2 B.1.1.7 (UK variant) and the novel B.1.5.2.5 lineage in Oyo State, Nigeria. Preprint at medRxiv https://doi.org/10.1101/2021.04.09.21255206 (2021).

19. Pereira, F. et al. Genomic surveillance activities unveil the introduction of the SARS-CoV-2 B.1.525 variant of interest in Brazil: case report.J. Med. Virol. 93, 5523-5526 (2021).

20. Edara, V. V. et al. Infection and vaccine-induced neutralizing antibody responses to the SARS-CoV-2 B.1.617.1 variant. Preprint at bioRxiv https://doi.org/10.1101/2021.05.09.443299 (2021).

21. Baum, A. et al. REGN-COV2 antibodies prevent and treat SARS-CoV-2 infection in rhesus macaques and hamsters. Science 370, 1110-1115 (2020).

22. Baum, A. et al. Antibody cocktail to SARS-CoV-2 spike protein prevents rapid mutational escape seen with individual antibodies. Science 369, 1014-1018 (2020).

23. Wang, N. et al. Structure-based development of human antibody cocktails against SARS-CoV-2. Cell Res. 31, 101-103 (2020).

24. Yao, H. et al. Rational development of a human antibody cocktail that deploys multiple functions to confer pan-SARS-CoVs protection. Cell Res. 31, 25-36 (2021).

25. Labrijn, A. F., Janmaat, M. L., Reichert, J. M. \& Parren, P. Bispecific antibodies: a mechanistic review of the pipeline. Nat. Rev. Drug Discov. 18, 585-608 (2019).

26. Demanet, C., Brissinck, J., De Jonge, J. \& Thielemans, K. Bispecific antibody-mediated immunotherapy of the BCL1 lymphoma: increased efficacy with multiple injections and CD28-induced costimulation. Blood 87, 4390-4398 (1996).

27. Li, J. F. \& et al. A novel bispecific c-MET/CTLA-4 antibody targetting lung cancer stem cell-like cells with therapeutic potential in human non-small-cell lung cancer. Biosci. Rep. 39, BSR20171278 (2019).

28. Lum, L. G. et al. Targeting cytomegalovirus-infected cells using T cells armed with anti-CD3 $\mathrm{x}$ anti-CMV bispecific antibody. Biol. Blood Marrow Transpl. 18, 1012-1022 (2012).
29. Wang, J. et al. A human bi-specific antibody against Zika virus with high therapeutic potential. Cell 171, 229-241 (2017).

30. De Gasparo, R. et al. Bispecific IgG neutralizes SARS-CoV-2 variants and prevents escape in mice. Nature 593, 424-428 (2021).

31. Bournazos, S., Gazumyan, A., Seaman, M. S., Nussenzweig, M. C. \& Ravetch J. V. Bispecific anti-HIV-1 antibodies with enhanced breadth and potency. Cell 165, 1609-1620 (2016).

32. Frei, J. C. et al. Bispecific antibody affords complete post-exposure protection of mice from both Ebola (Zaire) and Sudan viruses. Sci. Rep. 6, 19193 (2016).

33. Moshoette, T., Ali, S. A., Papathanasopoulos, M. A. \& Killick, M. A. Engineering and characterising a novel, highly potent bispecific antibody iMab-CAP256 that targets HIV-1. Retrovirology 16, 31 (2019).

34. Asokan, M. et al. Bispecific antibodies targeting different epitopes on the HIV-1 envelope exhibit broad and potent neutralization. J. Virol. 89, 12501-12512 (2015).

35. Zhou, W., Xu, C., Wang, P., Luo, M. \& Jiang, Q. N439K variant in spike protein may alter the infection efficiency and antigenicity of SARS-CoV-2 based on molecular dynamics simulation. Preprint at bioRxiv https://doi. org/10.1101/2020.11.21.392407 (2020)

36. Thomson, E. C. et al. Circulating SARS-CoV-2 spike N439K variants maintain fitness while evading antibody-mediated immunity. Cell 184, 1171-1187 e1120 (2021).

37. Hirotsu, Y. \& Omata, M. Discovery of a SARS-CoV-2 variant from the P.1 lineage harboring K417T/E484K/N501Y mutations in Kofu, Japan. J. Infect. 82, 276-316 (2021).

38. Chakraborty, S. Evolutionary and structural analysis elucidates mutations on SARS-CoV2 spike protein with altered human ACE2 binding affinity. Biochem. Biophys. Res. Commun. 538, 97-103 (2021).

39. Starr, T. N. et al. Prospective mapping of viral mutations that escape antibodies used to treat COVID-19. Science 371, 850-854 (2021).

40. Dejnirattisai, W. et al. Cross-reacting antibodies enhance dengue virus infection in humans. Science 328, 745-748 (2010).

41. Sridhar, S. et al. Effect of dengue serostatus on dengue vaccine safety and efficacy. N. Engl. J. Med. 379, 327-340 (2018).

42. Hohdatsu, T. et al. Antibody-dependent enhancement of feline infectious peritonitis virus infection in feline alveolar macrophages and human monocyte cell line U937 by serum of cats experimentally or naturally infected with feline coronavirus. J. Vet. Med Sci. 60, 49-55 (1998).

43. Wang, S. F. et al. Antibody-dependent SARS coronavirus infection is mediated by antibodies against spike proteins. Biochem. Biophys. Res. Commun. 451, 208-214 (2014).

44. Du, L. Y., Tai, W. B., Zhou, Y. S. \& Jiang, S. B. Vaccines for the prevention against the threat of MERS-CoV. Expert Rev. Vaccines 15, 1123-1134 (2016).

Publisher's note Springer Nature remains neutral with regard to jurisdictional claims in published maps and institutional affiliations.

(c) The Author(s), under exclusive licence to Springer Nature America, Inc. 2022 


\section{Methods}

Construction, expression and purification of bsAbs. bsAb13 and bsAb14 were engineered in the DVD-Ig format. The B38 variable domain of the heavy chain $\left(\mathrm{V}_{\mathrm{H}} 38\right)$ and $\mathrm{H} 4$ variable domain of the heavy chain $\left(\mathrm{V}_{\mathrm{H}} 4\right)$ were fused with a GGGGSGGGGS linker, followed by a constant region (CH1-CH2-CH3). For the light chain of bsAb13, the $\mathrm{B} 38$ variable domain $\left(\mathrm{V}_{\mathrm{L}} 38\right)$ and $\mathrm{H} 4$ variable domain $\left(\mathrm{V}_{\mathrm{L}} 4\right)$ were connected via the GGGGSGGGGS linker, followed by a constant region $\left(\mathrm{C}_{\mathrm{L}}\right)$. The positions of the variable regions of $\mathrm{B} 38$ and $\mathrm{H} 4$ are reversed in bsAb14 compared to bsAb13. BsAb15 and bsAb16 represent the IgG-ScFv format. Specifically, the $\mathrm{H} 4 \mathrm{ScFv}$ was connected to the $\mathrm{C}$ terminus of the B38 heavy chain by a GGS linker in the bsAb15 heavy chain. For bsAb16, the B38 $\mathrm{ScFv}$ was connected to the $\mathrm{C}$ terminus of the $\mathrm{H} 4$ heavy chain by a GGS linker. The light chains of B38 and H4 were paired with the heavy chains of bsAb15 and bsAb16, respectively.

The plasmids encoding the heavy chain and light chain were cotransfected into HEK293F cells. The molar ratio of heavy chain and light chain was $1: 1.5$, with a total of $2 \mu \mathrm{g}$ plasmid and $4 \mu \mathrm{g}$ Polyetherimide (PEI) $\left(1 \mathrm{mg} \mathrm{ml}^{-1}\right)$ per milliliter HEK293F cells. Cells were cultured with SMM 293-TII medium (Sinobiological) at $310 \mathrm{~K}$ and $5 \% \mathrm{CO}_{2}$, and they were supplemented with $35 \mathrm{ml} \mathrm{l}^{-1}$ SMS M293-SUPI (Sinobiological) at $24 \mathrm{~h}$ after transfection. Supernatants were collected on the fifth day, filtered through a $0.22-\mu \mathrm{m}$ membrane, purified over a Protein A HP column (GE Healthcare) and then further purified by size-exclusion chromatography using a Superdex 200 10/300 increase column (GE Healthcare) in PBS buffer (pH 7.4). The antibodies used in rhesus macaque protection assays, pharmacokinetics and $\mathrm{ADE}$ assays were produced in $\mathrm{CHO}$ cells (Wuxi Biopharmaceuticals).

Expression and purification of RBDs. The optimized coding sequence of the WT SARS-CoV-2 RBD (residues 319-541, GenBank: YP_009724390.1) with a C-terminal $6 \times$ His tag was cloned into the pCAGGS mammalian expression vector. Mutated RBDs (K417N, N460S, E484K, E484A and N501Y) were subcloned into pCAGGS. Plasmid $(2 \mu \mathrm{g})$ and PEI were transiently cotransfected at the ratio of 1:3 per milliliter HEK293F cells. Cells were cultured in SMM 293-TII medium (Sinobiological) at $310 \mathrm{~K}$ and $5 \% \mathrm{CO}_{2}$ and then supplemented with $35 \mathrm{ml} \mathrm{l}^{-1}$ SMS M293-SUPI (Sinobiological) at $24 \mathrm{~h}$ after transfection. Supernatants were collected on the fifth day, filtered through a $0.22-\mu \mathrm{m}$ membrane, purified over a His-trap HP column (GE Healthcare) and then further purified by size-exclusion chromatography using a Superdex 200 increase column (GE Healthcare) in PBS buffer (pH 7.4).

Competition assay. Biotinylated SARS-CoV-2 RBD $\left(10 \mu \mathrm{g} \mathrm{ml}^{-1}\right)$ was immobilized on streptavidin sensors and saturated with $500 \mathrm{nM} \mathrm{B} 38$ or H4, followed by $500 \mathrm{nM}$ $\mathrm{bsAb}$. The data were analyzed using biolayer interferometry data analysis software. The buffer for all proteins was $20 \mathrm{mM}$ HEPES, pH 7.4, $150 \mathrm{mM} \mathrm{NaCl}$ and $0.05 \%$ (v/v) Tween 20 .

SPR. The affinity between bsAbs and SARS-CoV-2 RBD was measured using a Biacore $8 \mathrm{~K}$ system. BsAbs were immobilized on protein A chips, and serially diluted SARS-CoV-2 RBD was added. All of the proteins were in a buffer composed of $20 \mathrm{mM}$ HEPES, $\mathrm{pH} 7.4,150 \mathrm{mM} \mathrm{NaCl}$ and $0.05 \%$ (v/v) Tween 20 . A two-state reaction model was used to analyze data with the Biacore evaluation software.

Pseudovirus neutralization. The replication-deficient vesicular stomatitis virus vector backbone (VSV- $\Delta \mathrm{G}-\mathrm{GFP}$ ) plasmid and the corresponding S plasmid with an 18-residue deletion were cotransfected into HEK293T cells to generate pseudoviruses as described previously ${ }^{45}$. The initial concentrations of the B38 to WT, N460S mutant and Delta variant pseudoviruses were $100 \mu \mathrm{g} \mathrm{ml}^{-1}$, $1,600 \mu \mathrm{g} \mathrm{ml}^{-1}$ and $5 \mu \mathrm{g} \mathrm{ml}^{-1}$. The initial concentrations of the bsAb15 to WT, Alpha and Delta variant pseudoviruses were $6.25 \mu \mathrm{g} \mathrm{ml}^{-1}, 12.5 \mu \mathrm{g} \mathrm{ml}^{-1}$ and $1 \mu \mathrm{g} \mathrm{ml}^{-1}$. The initial concentrations of the B38 to mutant pseudoviruses (K417N, E484K, E484A, Alpha, Beta, Kappa and Eta variants) were $200 \mu \mathrm{g} \mathrm{ml}^{-1}$. The initial concentrations of the bsAb15 to mutant pseudoviruses (K417N, N460S, E484K, E484A, Beta, Kappa and Eta) were $200 \mu \mathrm{g} \mathrm{ml}^{-1}$. The initial concentration of the H4 to Delta variant pseudovirus was $5 \mu \mathrm{g} \mathrm{ml}^{-1}$. The initial concentrations of the $\mathrm{H} 4$ to other mutant pseudoviruses mentioned above were $200 \mu \mathrm{g} \mathrm{ml}^{-1}$. The initial concentration of the B38, H4, B38 + H4 cocktail and bsAb15 to Q474H pseudoviruses was $400 \mu \mathrm{g} \mathrm{ml}^{-1}$. The initial concentrations of bsAb13, bsAb14 and bsAb16 were $100 \mu \mathrm{g} \mathrm{ml}^{-1}, 100 \mu \mathrm{g} \mathrm{ml}^{-1}$ and $6.25 \mu \mathrm{g} \mathrm{ml}^{-1}$. For each antibody, triplicates were set up, and an equal volume of antibody dilutions was mixed with pseudoviruses at $310 \mathrm{~K}$ for $30 \mathrm{~min}$. The mixture was then added into Vero E6 cells for $15 \mathrm{~h}$ at $310 \mathrm{~K}$ in $5 \%$ $\mathrm{CO}_{2}$. Wells with pseudovirus only were used as controls. Data were measured using a CQ1 quantitative confocal image cytometer (Yokogawa), and $\mathrm{IC}_{50}$ values were analyzed with GraphPad Prism 8.0 software.

Authentic virus neutralization. Vero E6 cells were seeded in 96-well plates pre-prepared 1 day before use and grown to approximately $85 \%$ confluence in these assays. Antibodies were serially diluted in DMEM media with starting concentrations of $50 \mu \mathrm{g} \mathrm{ml}^{-1}(\mathrm{~B} 38, \mathrm{H} 4, \mathrm{~B} 38+\mathrm{H} 4$, bsAb13, bsAb15 and bsAb16) and $100 \mu \mathrm{g} \mathrm{ml}^{-1}$ (bsAb14), respectively. Ten gradients with eight replicates analyzed using the assay. Virus $\left(50 \mu \mathrm{l}\right.$ of $2 \times 10^{3} \mathrm{TCID}_{50} \mathrm{ml}^{-1}$
SARS-CoV-2; hCoV-19/China/CAS-B001/2020, National Microbiology Data Center NMDCN0000102-3, GISAID databases EPI_ISL_514256-7) was mixed with $50 \mu \mathrm{l}$ antibody dilutions and incubated at $310 \mathrm{~K}$ for $1 \mathrm{~h}$. Then, $100 \mu \mathrm{l}$ of the mixture was added per well, and the virus control and negative control were $2 \times 10^{3} \mathrm{TCID}_{50} \mathrm{ml}^{-1}$ authentic virus and diluted DMEM, respectively. After culturing at $310 \mathrm{~K}$ and $5 \% \mathrm{CO}_{2}$ for 3 days, CPE was observed. $\mathrm{IC}_{50}$ values were calculated with GraphPad Prism 8.0 software. For the Delta virus neutralization assay, the initial concentrations of B38, cocktail, $\mathrm{H} 4$ and bsAb15 were $400 \mathrm{nM}, 400 \mathrm{nM}, 1,600 \mathrm{nM}$ and $50 \mathrm{nM}$, respectively.

Escape mutant screening and sequencing. Escape mutants were selected under pressure of individual mAbs or a mAb cocktail, as well as bsAb, using the SARS-CoV-2 virus. The experiments were performed as described by Alina et al. ${ }^{22}$, with some modifications. Briefly, a total of $1 \times 10^{3}$ plaque-forming units of the parental SARS-CoV-2 was incubated with a series of twofold dilutions (ranging from $500 \mu \mathrm{g} \mathrm{ml}^{-1}$ to $1.95 \mu \mathrm{g} \mathrm{ml}^{-1}$ ) of each antibody for $1 \mathrm{~h}$ and then added to a monolayer of Vero E6 cells. The supernatant was discarded after 1-h incubation and cells were washed with PBS three times, and then the media containing the diluted Abs were added to the wells. The development of CPE was monitored over 4 days, and supernatants were collected from wells with the highest concentration of antibody with detectable CPE ( $\geq 20 \% \mathrm{CPE}$ ). For a second-round passage, $200 \mu$ l of the passage 1 supernatant was expanded in a T25 flask containing Vero E6 cells under the same antibody pressure. For the third-round passage, which was the same as the first round, $200 \mu \mathrm{l}$ of the passage 2 supernatant was expanded for 4 days under increasing antibody selection in fresh Vero E6 cells. Viral RNA from wells with the highest $\mathrm{mAb}$ concentration and detectable $\mathrm{CPE}$ on passage 1 or 3 (collected 4 days after infection) was isolated with the QIAamp Viral RNA mini kit (QIAGEN). cDNA synthesis and library preparation were performed using the KAPA RNA HyperPrep Kit Illumina Platforms. Random primers were used for amplifying the genome sequence of SARS-CoV-2. The RNA-sequencing data were acquired by next-generation sequencing technology using Illumina NovaSeq 6000 . Raw next-generation sequencing reads were processed by filtering sequence length $<50$ and $\mathrm{N}$ base number $>6$ with fastp software (version 0.20 .0$)\left(\right.$ ref. $\left.{ }^{46}\right)$. Then, the filtered reads were mapped to SARS-CoV-2 virus by Burrows-Wheeler aligner (BWA version 0.7.17-r1188) (ref. ${ }^{47}$ ). SAMtools (version 1.9) was used for SNP calling with default settings ${ }^{48}$. Single-nucleotide polymorphisms with a minimum frequency of $5 \%$ were identified and annotated.

Prophylaxis and treatment in mice. Prophylaxis (study A) and treatment (study B) studies were performed in a Ad5-hACE2 infection BALB/c mice model (6-week-old females). Briefly, $1.6 \times 10^{11} \mathrm{vp} \mathrm{m}^{-1}$ replication-deficient Ad5-hACE2 was intranasally injected at day 5 . In the prophylaxis group, $50 \mathrm{mg} \mathrm{kg}^{-1} \mathrm{bsAb} 15$ was intraperitoneally injected at $24 \mathrm{~h}$, and the mice were challenged with a dose of $2 \times 10^{6} \mathrm{TCID}_{50}$ per milliliter SARS-CoV-2. In the treatment group, two doses of $\mathrm{Ab} 15$ (25 mg kg-1 and $50 \mathrm{mg} \mathrm{kg}^{-1}$ ) and one dose of parental mAb (B38, H4 or cocktail) were intraperitoneally injected at $12 \mathrm{~h}$ after challenge in each group. Mice were executed and necropsied 3 days after challenge with virus. Mice were treated with PBS in the control group. The amount of RNA copies per gram of tissue was determined using a RT-qPCR assay. Viral RNA was extracted from lungs using a QIAamp Viral RNA mini kit (Qiagen), and qPCR assays were performed using a COVID-19 virus Dual-Detection Kit (MABSKY) on a QuantStudio 7 Real-Time PCR System (Applied Biosystems) and amplified using the following program: $50^{\circ} \mathrm{C}$ for $30 \mathrm{~min}, 95^{\circ} \mathrm{C}$ for $3 \mathrm{~min}$ and 45 cycles of $95^{\circ} \mathrm{C}$ for $5 \mathrm{~s}$ and $55^{\circ} \mathrm{C}$ for $30 \mathrm{~s}$. The number of copies of RNA per gram was calculated by extrapolation from the standard curve. Lung sections were collected, fixed in $4 \%$ formaldehyde solution and embedded in paraffin to prepare for H\&E staining. Slides were blindly evaluated by board-certified veterinary pathologists. Whole digital slide images were screened and analyzed using HALO Version3.2. The animals used in the experiments were allocated at random. Data collection and analysis were not performed blind to the conditions of the experiments. No statistical methods were used to predetermine sample sizes, but our sample sizes are similar to those reported in previous publications ${ }^{49,50}$. No animals or data points were excluded from the analyses. The mouse room has a 12-h light/dark cycle. The room temperature is $20-24^{\circ} \mathrm{C}$, and the humidity is around $50 \%$.

Protection in rhesus macaques. Rhesus macaques $(1.5-2.5 \mathrm{~kg})$ were divided into four groups for placebo, prophylaxis $\left(50 \mathrm{mg} \mathrm{kg}^{-1}\right)$, low-dose treatment $\left(25 \mathrm{mg} \mathrm{kg}^{-1}\right)$ and high-dose treatment $\left(50 \mathrm{mg} \mathrm{kg}^{-1}\right)$, respectively. For the prophylaxis group, each rhesus macaque was intravenously administered with one dose of $50 \mathrm{mg} \mathrm{kg}^{-1}$ bsAb15 and challenged intranasally $12 \mathrm{~h}$ later with $100 \mu \mathrm{l} 5.0 \operatorname{lgCCID}_{50}$ SARS-CoV-2. For the treatment group, each rhesus macaque was challenged intranasally $12 \mathrm{~h}$ before the first treatment with $100 \mu \mathrm{l}$

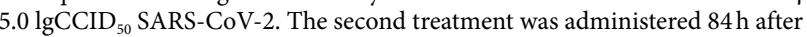
infection. Throat and nasal swab samples were collected daily from day 1 to day 7. RNA was extracted using a Takara RNA extraction kit for qPCR with primers for E, ORF1ab and sgRNA. The following primer sequences were used: ORF1ab forward, 5' -CCCTGTGGGTTTTACACTTAA-3'; ORF1ab reverse, $5^{\prime}$-ACGATTGTGCATCAGCTGA-3' ${ }^{\prime}$; probe (FAM-TAMRA), $5^{\prime}$-CCGTCTGCGG TATGTGGAAAGGTTATGG-3' ${ }^{\prime}$; sgRNA forward, $5^{\prime}$-CGATCTCTTGTAGATCT

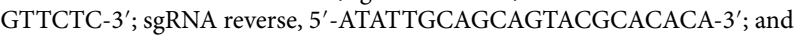


probe (FAM-TAMRA), 5' -ACACTAGCCATCCTTACTGCGCTTCG-3'. All rhesus macaques were killed and necropsied at $7 \mathrm{dpi}$. Lungs were fixed in $4 \%$ formaldehyde solution. The fixed lung tissues were embedded in paraffin, sectioned and stained with H\&E for histopathological analysis.

Pharmacokinetics in Macaca fascicularis. The pharmacokinetics assays were performed by JOINN Laboratories. Specifically, 18 Macaca fascicularis (nine males and nine females, 2.8-3.7 years old) were divided into three groups, which were administered with three different dosages of bsAb15 $\left(7.5 \mathrm{mg} \mathrm{kg}^{-1}, 25 \mathrm{mg} \mathrm{kg}^{-1}\right.$ and $75 \mathrm{mg} \mathrm{kg}^{-1}$ ) intravenously. Blood samples were collected from all monkeys before dosing and $10 \mathrm{~min}, 23 \mathrm{~min}$ ( $3 \mathrm{~min}$ after the completion of dosing), $2 \mathrm{~h}, 4 \mathrm{~h}, 8 \mathrm{~h}, 24 \mathrm{~h}$ (day 2), $48 \mathrm{~h}$ (day 3), $72 \mathrm{~h}$ (day 4), 96h (day 5), $168 \mathrm{~h}$ (day 8), 336h (day 15), $504 \mathrm{~h}$ (day 22 ) and $672 \mathrm{~h}$ (day 29) after the start of dosing. Blood (approximately $0.8 \mathrm{ml}$ ) was collected from a hindlimb subcutaneous vein of monkeys. The centrifuge tubes coated with separation gel and coagulation were stored in room temperature before use. The collected blood samples were transferred into these labeled centrifuge tubes and be stored and transferred at room temperature and then were centrifuged at $1,500 \mathrm{~g}$ for $10 \mathrm{~min}$ at room temperature within $2 \mathrm{~h}$ after collection

Sera samples were collected at $10 \mathrm{~min}, 23 \mathrm{~min}, 2 \mathrm{~h}, 4 \mathrm{~h}, 8 \mathrm{~h}, 24 \mathrm{~h}, 48 \mathrm{~h}, 72 \mathrm{~h}, 96 \mathrm{~h}$, $168 \mathrm{~h}, 336 \mathrm{~h}, 504 \mathrm{~h}$ and $672 \mathrm{~h}$ after bsAb15 administration, and the concentration of bsAb15 in each sample was detected by enzyme-linked immunosorbent assay. The concentration of bsAb15 in serum was detected by enzyme-linked immunosorbent assay with SARS-CoV-2 (COVID-19) S protein RBD proteins with mouse IgG2a Fc tag $\left(2 \mu \mathrm{g} \mathrm{ml}^{-1}\right)$ precoated on the microplate wells. The bound bsAb15 proteins were then detected with goat anti-human IgG, monkey ads-horseradish peroxidase. The shade of color associates with the concentration of bsAb15 in given range. Serially diluted bsAb15 proteins with blank monkey serum with the concentration of $800 \mathrm{ng} \mathrm{ml}^{-1}, 400 \mathrm{ng} \mathrm{ml}^{-1}, 300 \mathrm{ng} \mathrm{ml}^{-1}, 200 \mathrm{ng} \mathrm{ml}^{-1}, 100 \mathrm{ng} \mathrm{ml}^{-1}, 50.0 \mathrm{ng} \mathrm{ml}^{-1}$, $25.0 \mathrm{ng} \mathrm{ml}^{-1}$ and $12.5 \mathrm{ng} \mathrm{ml}^{-1}$ were enrolled as calibration curve samples, with blank monkey serum as a blank control. Data were analyzed using a Watson LIMS 7.5 system and WinNonlin software 8.0.0.3176.

ADE of viral entry. CD32A H131-CHOK1 cells $\left(5 \times 10^{5}\right.$ cells per milliliter) (GenScript, M00598) were seeded into 96-well plates. An equal volume of SARS-CoV-2 pseudovirus $\left(1.3 \times 10^{4} \mathrm{TCID}_{50}\right.$ per milliliter; NIFDC, catalog number 20200603 ) and diluted bsAb15 (final concentration ranging from 10,000 to $0.013 \mathrm{ng} \mathrm{ml}^{-1}$ ) were incubated at $310 \mathrm{~K}$ in $5 \% \mathrm{CO}_{2}$ for $1 \mathrm{~h}$ and then added to the cells. Wells containing cells and pseudovirus were used as negative controls. A mixture of two antibodies (AS35 and AS36) was used as a positive control (WuXi Biologics, catalog numbers SAD-S35 and SA2-S36). After incubation for 20-24h, the supernatants were discarded and filled with britelite-plus chemical reagent (PerkinElmer). The plates were shaken in the dark for 3-6 min. Cells were then blended and transferred into white 96-well plates. Luminescence values were measured with a microplate reader (Envision). Data were analyzed with SoftMax Pro software.

Ethics statement. This study was performed in strict accordance with the recommendations in the Guide for the Care and Use of Laboratory Animals of the Institute of Microbiology, Chinese Academy of Sciences Ethics Committee, Institute of Medical Biology, Chinese Academy of Medical Sciences and JOINN (Suzhou) Institutional Animal Care and Use Committee. All experiments conform to the relevant regulatory standards. All animal experiments were conducted under isoflurane anesthesia to minimize animal suffering. Mice and monkey studies with authentic SARS-CoV-2 were conducted under animal BSL3 (A-BSL3) containment.

Reporting Summary. Further information on research design is available in the Nature Research Reporting Summary linked to this article.

\section{Data availability}

All data are present in the article and Supplementary Information files or from the corresponding author upon reasonable request. Source data are provided as Source Data files. Source data are provided with this paper.

\section{References}

45. Li, Q. et al. The impact of mutations in SARS-CoV-2 spike on viral infectivity and antigenicity. Cell 182, 1284-1294 (2020).

46. Chen, S., Zhou, Y., Chen, Y. \& Gu, J. fastp: an ultra-fast all-in-one FASTQ preprocessor. Bioinformatics 34, i884-i890 (2018).

47. Li, H. \& Durbin, R. Fast and accurate short read alignment with Burrows-Wheeler transform. Bioinformatics 25, 1754-1760 (2009).

48. Li, H. et al. The Sequence Alignment/Map format and SAMtools. Bioinformatics 25, 2078-2079 (2009).

49. Wang, Q. et al. Neutralization mechanism of human monoclonal antibodies against Rift Valley fever virus. Nat. Microbiol 4, 1231-1241 (2019).

50. Jiang, H. et al. Structure-based modification of an anti-neuraminidase human antibody restores protection efficacy against the drifted influenza virus. mBio. 11, e02315-20 (2020)

\section{Acknowledgements}

This work was supported by the National Natural Science Foundation of China (82022042, 32090014 and 31872745), the National Key R\&D Program of China (2020YFA0907104) and the intramural special grant for SARS-CoV-2 research from the Chinese Academy of Sciences.

\section{Author contributions}

Y.W., F.G., G.F.G. and S.T. initiated and coordinated the project and designed the experiments. Z.L., G.Z., Z.C., X.Zhang, J.Z., F.W., W.Z., Y.C. and L.S. expressed and purified the proteins. Z.L. and G.Z. conducted the SPR and Octet analyses with the help of Z.F. Z.L. and G.Z. evaluated the neutralizing potency with help from X.Zhao, D.L. and R.Z.. S.L., W.P. and Z.H. conducted the animal experiments. J.H. analyzed the H\&E staining data. RT-qPCR was conducted by S.L., W.P. and Z.H. K.X., W.L., G.W. and P.L. operated and coordinated the authentic virus neutralization assays. Y.W. wrote the manuscript, and Y.W., F.G., S.T., S.L. and G.F.G. revised the manuscript.

\section{Competing interests}

Z.L., S.L., S.T., Y.W. and G.F.G. are listed as inventors on pending patent applications for bsAb15. The other authors declare no competing interests.

\section{Additional information}

Extended data is available for this paper at https://doi.org/10.1038/s41590-022-01138-w. Supplementary information The online version contains supplementary material available at https://doi.org/10.1038/s41590-022-01138-w.

Correspondence and requests for materials should be addressed to Shuguang Tan, George Fu Gao, Feng Gao or Yan Wu.

Peer review information Nature Immunology thanks Wayne Marasco and the other, anonymous, reviewer(s) for their contribution to the peer review of this work. N. Bernard was the primary editor on this article and managed its editorial process and peer review in collaboration with the rest of the editorial team.

Reprints and permissions information is available at www.nature.com/reprints. 
A

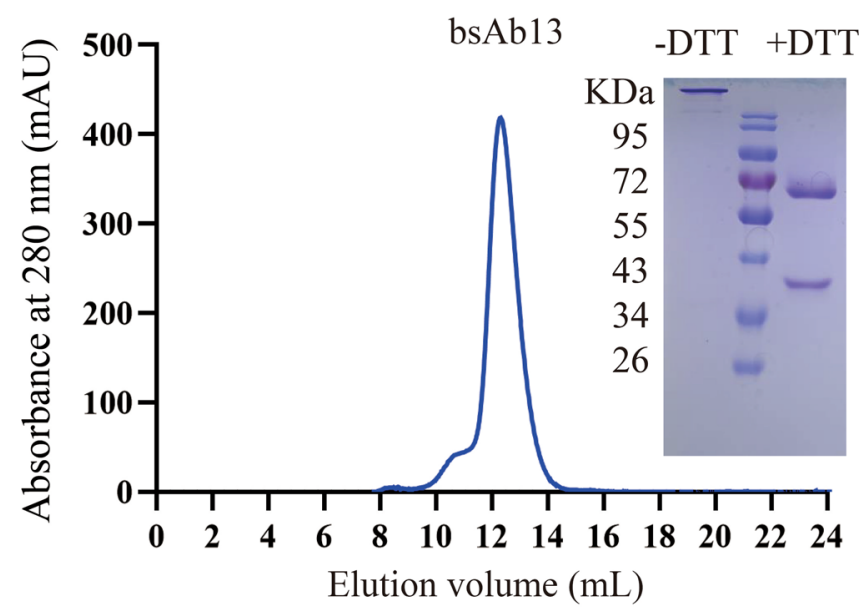

C

ఏ్త
bsAb15

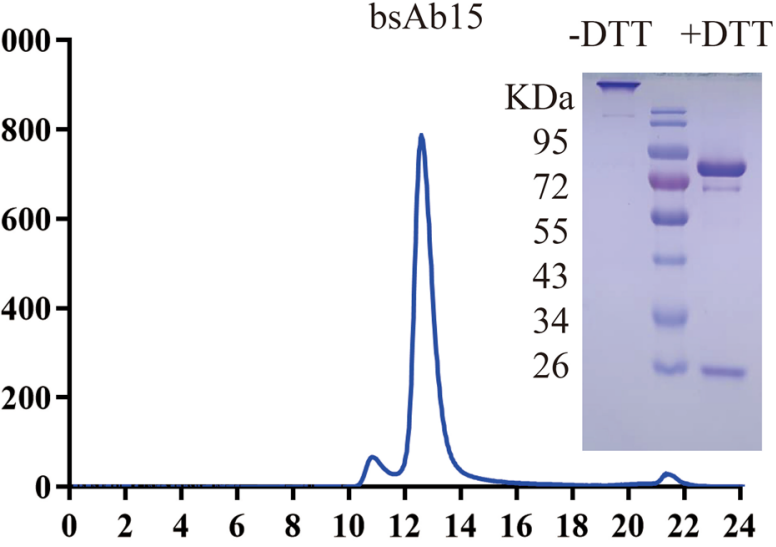

Elution volume $(\mathrm{mL})$
B

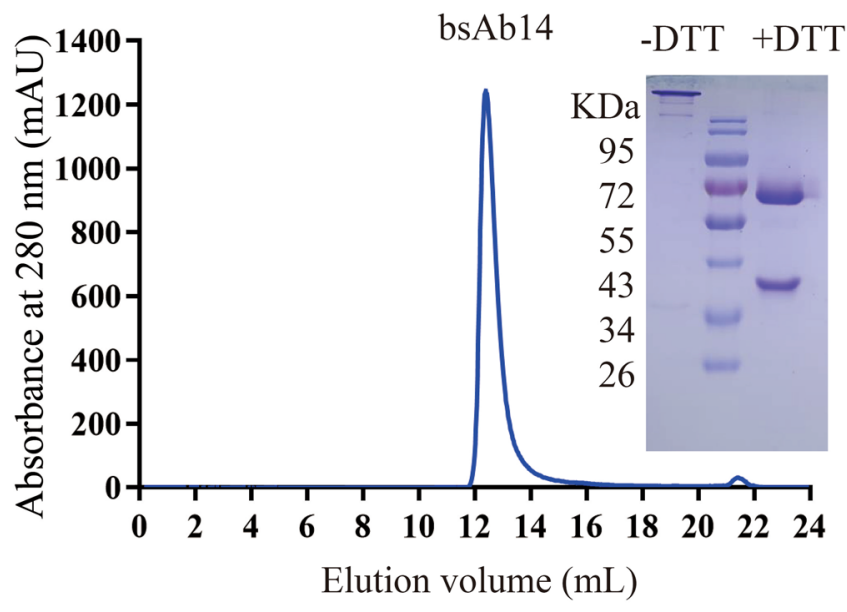

D

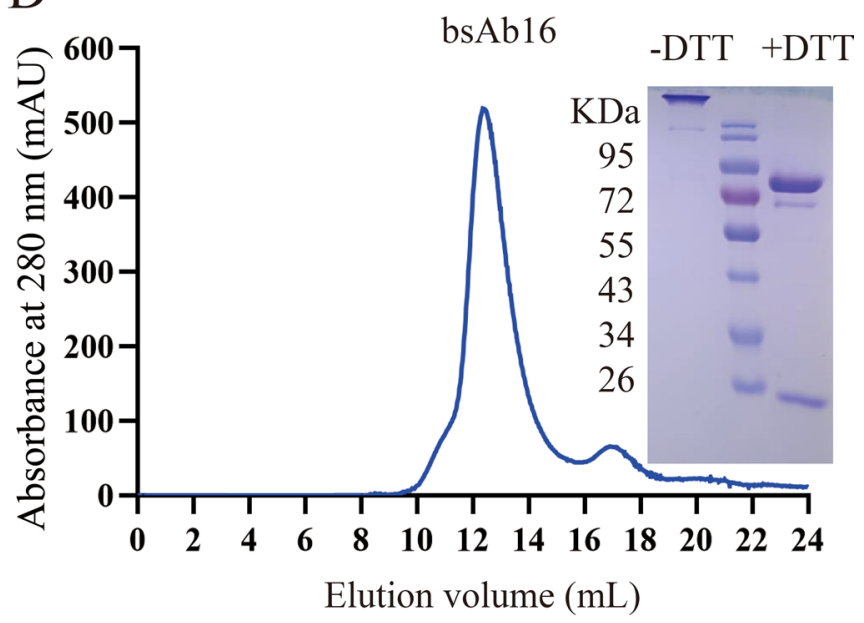

Extended Data Fig. 1 | Size-exclusion analysis of bsAbs. (a to d) The bsAbs were loaded on a Superdex 200 increase column individually. The SDS-PAGE profiles of the pooled samples under reducing and non-reducing conditions. The heavy and light chains of bsAb13 and bsAb14 are about 65 KDa and 40 KDa, respectively, while the heavy and light chains of bsAb15 and bsAb16 are $80 \mathrm{KDa}$ and $25 \mathrm{KDa}$, respectively. 


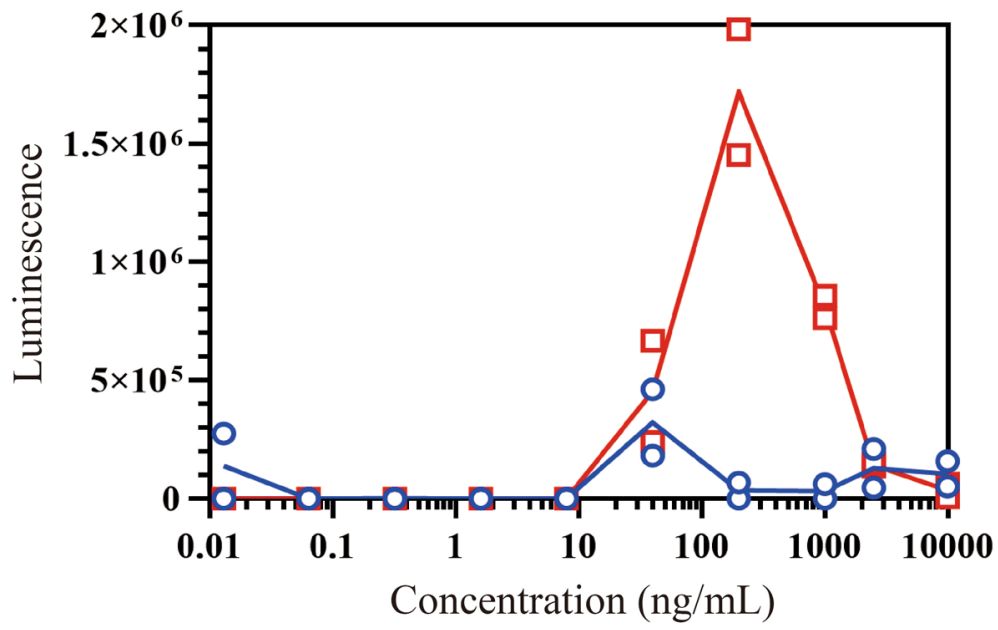

Extended Data Fig. 2 | The result of ADE viral entry of bsAb15 evaluated by CD32A H131-CHOK1 cell system. The luminescence values of wells containing different concentrations of bsAb15 (blue), and positive control (red) indicated the ADE viral entry level. 

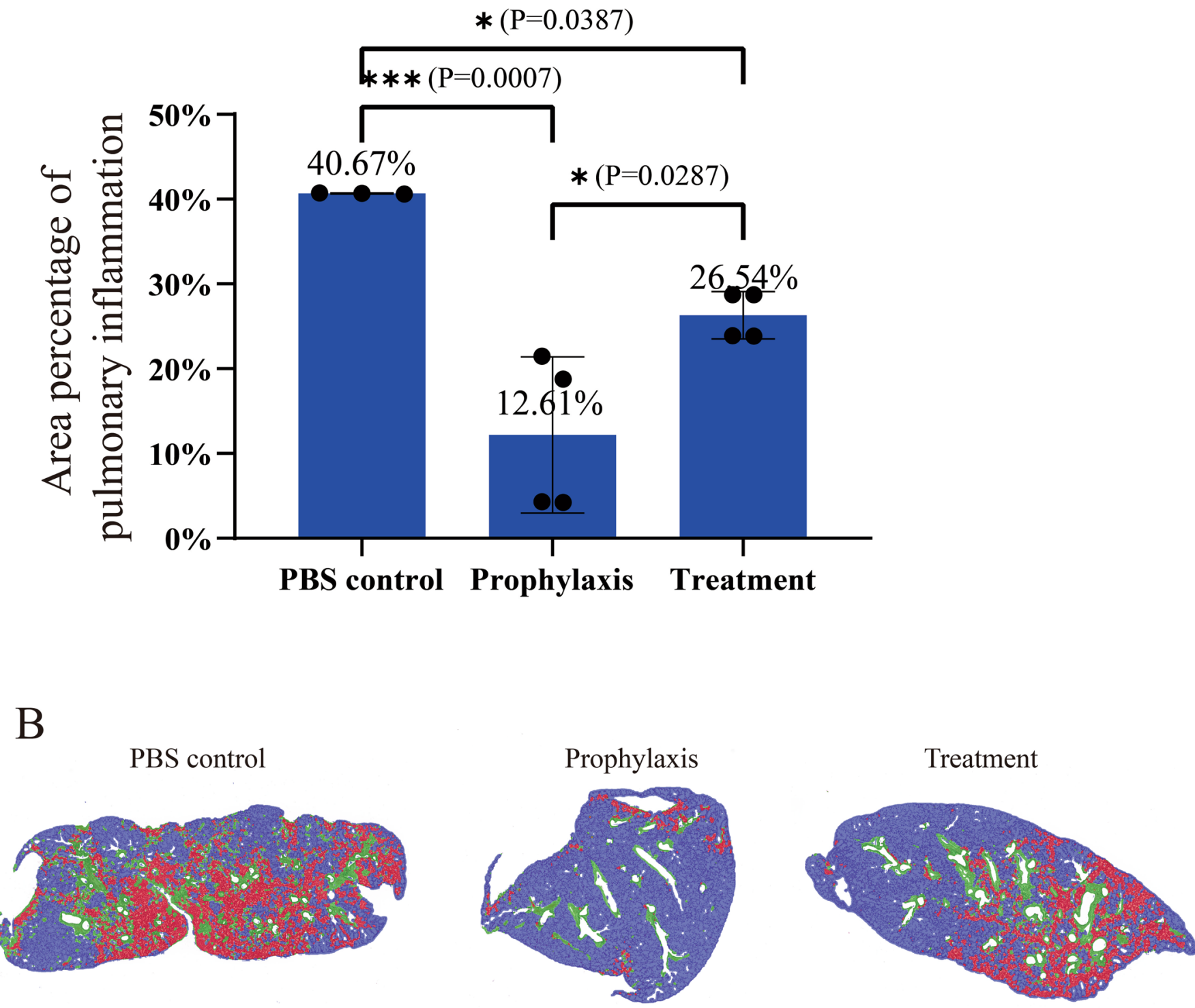

$1 \mathrm{~mm}$

$1 \mathrm{~mm}$

$1 \mathrm{~mm}$

Extended Data Fig. 3 | The quantitative analysis of pulmonary inflammation of lung samples from PBS control group, prophylaxis group and treatment group. (a) The area percentage of pulmonary inflammation were estimated by pathological quantitative analysis using Halo v3.2 Classifier DenseNet Al (Plugin) in three groups $(n \geq 3)$. Data are shown as the means with SD and were analyzed by one-way ANOVA, ${ }^{\star} p<0.05$ and ${ }^{\star \star \star} p<0.001$. (b) The models of pathological quantitative analysis in three groups. In the process of establishing the quantitative analysis algorithm, different types of tissue were represented by different colors. Normal lung, normal structural collagen and inflammation were labeled in blue, green and red, respectively. 


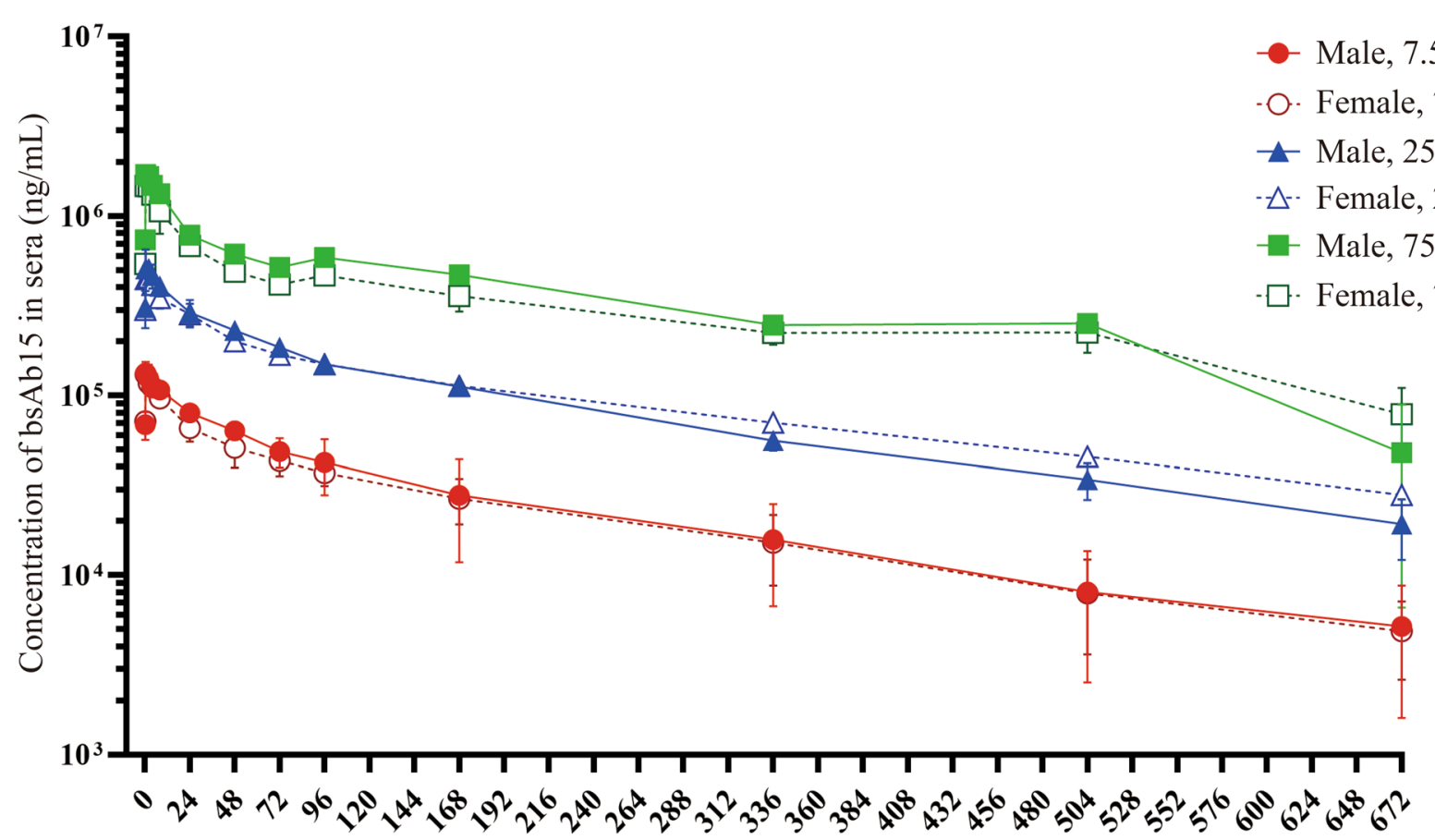

Time (h)

Extended Data Fig. 4 | Pharmacokinetics in Macaca fascicularis. Three dosages of bsAb15 were administered to male and female macaca fascicularis, respectively. The average level of bsAb15 in the sera of male groups and female groups were indicated as solid line and dotted line, respectively. Data are shown as the means with SD. 


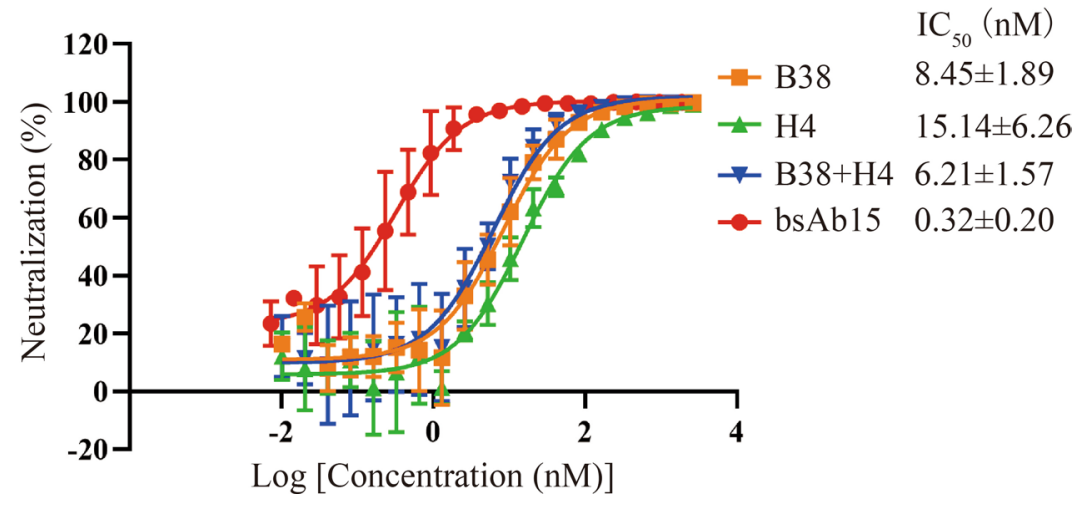

Extended Data Fig. 5 | Neutralization of antibodies against pseudovirus carrying Q474H mutation. B38, H4, cocktail and bsAb15 were analyzed using the VSV-based pseudovirus system. All of the experiments were performed three times $(n=3)$, and the IC50 was determined by log (inhibitor)-response of nonlinear regression and displayed as the means \pm SD. 


\section{Reporting Summary}

Nature Portfolio wishes to improve the reproducibility of the work that we publish. This form provides structure for consistency and transparency in reporting. For further information on Nature Portfolio policies, see our Editorial Policies and the Editorial Policy Checklist.

\section{Statistics}

For all statistical analyses, confirm that the following items are present in the figure legend, table legend, main text, or Methods section.

$\mathrm{n} / \mathrm{a} \mid$ Confirmed

$\bigotimes$ The exact sample size $(n)$ for each experimental group/condition, given as a discrete number and unit of measurement

$\square$ \ A statement on whether measurements were taken from distinct samples or whether the same sample was measured repeatedly

The statistical test(s) used AND whether they are one- or two-sided

Only common tests should be described solely by name; describe more complex techniques in the Methods section.

\ A description of all covariates tested

$\square$ A description of any assumptions or corrections, such as tests of normality and adjustment for multiple comparisons

A full description of the statistical parameters including central tendency (e.g. means) or other basic estimates (e.g. regression coefficient)

AND variation (e.g. standard deviation) or associated estimates of uncertainty (e.g. confidence intervals)

For null hypothesis testing, the test statistic (e.g. $F, t, r$ ) with confidence intervals, effect sizes, degrees of freedom and $P$ value noted

Give $P$ values as exact values whenever suitable.

Х $\square$ For Bayesian analysis, information on the choice of priors and Markov chain Monte Carlo settings

$\square$ For hierarchical and complex designs, identification of the appropriate level for tests and full reporting of outcomes

Х $\square$ Estimates of effect sizes (e.g. Cohen's $d$, Pearson's $r$ ), indicating how they were calculated

\section{Our web collection on statistics for biologists contains articles on many of the points above.}

\section{Software and code}

Policy information about availability of computer code

Data collection SPR binding experiments were carried out using a BIAcore 8K system (GE Healthcare). qPCR data were collected by Applied Biosystems QuantStudio 7 Flex Real-Time PCR systems. The RNA seq data were collected by Illumina Novaseq 6000 . The competition assays were performed by OCTET RED96. The neutralization assays by using pseudo virus were carried out using Confocal Quantitative Image Cytometer (CQ1).

Data analysis Biacore evaluation software version 4.1, Graph Pad Prism 8.0 software, Watson LIMS 7.5 system and WinNonlin software 8.0.0.3176, SoftMax Pro software version 6.3, fastp software (version 0.20.0), Burrows-Wheeler Aligner (BWA version 0.7.17-r1188), HALO Version3.2 and SAMtools (version: 1.9) were used.

For manuscripts utilizing custom algorithms or software that are central to the research but not yet described in published literature, software must be made available to editors and

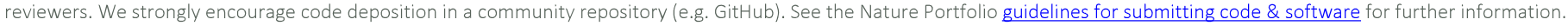

\section{Data}

Policy information about availability of data

All manuscripts must include a data availability statement. This statement should provide the following information, where applicable:

- Accession codes, unique identifiers, or web links for publicly available datasets

- A description of any restrictions on data availability

- For clinical datasets or third party data, please ensure that the statement adheres to our policy 
Please select the one below that is the best fit for your research. If you are not sure, read the appropriate sections before making your selection.

$\bigotimes$ Life sciences $\quad \square$ Behavioural \& social sciences $\square$ Ecological, evolutionary \& environmental sciences

For a reference copy of the document with all sections, see nature.com/documents/nr-reporting-summary-flat.pdf

\section{Life sciences study design}

All studies must disclose on these points even when the disclosure is negative.

Sample size No statistical test was used to determine the number of samples. Sample sizes were determined as appropriate to evaluate detection of animal experiments based on several studies done by us and others (mBio, 11 (5): e023115-20; Nature Microbiology, 4 (7): 1231-1241).

Data exclusions No data were excluded from the analyses

Replication All the experiments replicated twice at least other than neutralization of authentic Delta virus, and the sample sizes were presented in the figure legends

Randomization The animals used in experiments were allocated in random. For experiments without animals, randomization was not relevant.

Blinding Blinding was not completed, because the assays were done by the same group members.

\section{Reporting for specific materials, systems and methods}

We require information from authors about some types of materials, experimental systems and methods used in many studies. Here, indicate whether each material, system or method listed is relevant to your study. If you are not sure if a list item applies to your research, read the appropriate section before selecting a response.

Materials \& experimental systems

$\mathrm{n} / \mathrm{a}$ Involved in the study

$\square$ \ Antibodies

$\square \bigotimes$ Eukaryotic cell lines

\ $\square$ Palaeontology and archaeology

$\square \bigotimes$ Animals and other organisms

$\square$ Human research participants

\ $\square$ Clinical data

$\square \bigotimes$ Dual use research of concern

\section{Antibodies}

Antibodies used

Validation

B38, H4, bsAb13, bsAb14, bsAb15, bsAb16 are produced by the authors.

B38, H4, bsAb13, bsAb14, bsAb15, bsAb16 are human IgG1 antibodies. All of these mAbs are produced by HEK293F cells.

\section{Eukaryotic cell lines}

Policy information about cell lines

Cell line source(s)

HEK293F(ATCC CRL-1573), HEK293T(ATCC CRL-3216),Vero E6(ATCC CRL-1586), CD32A H131-CHOK1(GenScript, M00598),

Authentication

Mycoplasma contamination

Commonly misidentified lines

(See ICLAC register) CHO(ATCC PTA-9816)

None of the cell lines used are authenticated.

\author{
Methods \\ $\mathrm{n} / \mathrm{a}$ Involved in the study \\ X $\square$ ChIP-seq \\ Х $\square$ Flow cytometry \\ Х $\square$ MRI-based neuroimaging
}

All cell lines tested negative for mycoplasma contamination.

No misidentified lines are used. 
Policy information about studies involving animals; ARRIVE guidelines recommended for reporting animal research

Laboratory animals

45 six-week-old female Ad5-hACE2 infection BALB/c mice, 16 male 1.5-1.7 years-old rhesus macaque. 18 macaca fascicularis (9 male and 9 female). The mouse room has a 12 -hour light-dark cycle. The room temperature is $20-24^{\circ} \mathrm{C}$, and the humidity is around $50 \%$.

Wild animals

This study did not involve wild animals

Field-collected samples

Ethics oversight
The study did not involve the samples collected from field

This study was performed in strict accordance with the recommendations in the Guide for the Care and Use of Laboratory Animals of the Institute of Microbiology, Chinese Academy of Sciences (IMCAS) Ethics Committee, Institute of Medical Biology, Chinese Academy of Medical Sciences, and JOINN (Suzhou) Institutional Animal Care and Use Committee. All experiments conform to the relevant regulatory standards. All animal experiments were conducted under isoflurane anesthesia to minimize animal suffering. Mice and monkey studies with authentic SARS-CoV-2 were conducted under animal BSL3 (A-BSL3) containment

Note that full information on the approval of the study protocol must also be provided in the manuscript.

\section{Dual use research of concern}

Policy information about dual use research of concern

\section{Hazards}

Could the accidental, deliberate or reckless misuse of agents or technologies generated in the work, or the application of information presented in the manuscript, pose a threat to:
No $\mid$ Yes
$\square$ Public health
Х National security
Х $\square$ Crops and/or livestock
\ $\square$ Ecosystems
$\bigotimes \mid \square$ Any other significant area

$$
\text { Hazards SARS-CoV-2 }
$$

For examples of agents subject to oversight, see the United States Government Policy for Institutional Oversight of Life Sciences Dual Use Research of Concern.

\section{Experiments of concern}

Does the work involve any of these experiments of concern:
No $\mid$ Yes
Х Demonstrate how to render a vaccine ineffective
$\square \bigotimes$ Confer resistance to therapeutically useful antibiotics or antiviral agents
\ $\square$ Enhance the virulence of a pathogen or render a nonpathogen virulent
$\bigotimes \square$ Increase transmissibility of a pathogen
Х $\square$ Alter the host range of a pathogen
$\triangle \square$ Enable evasion of diagnostic/detection modalities
\ $\square$ Enable the weaponization of a biological agent or toxin
Х $\square$ Any other potentially harmful combination of experiments and agents

\section{Precautions and benefits}

Biosecurity precautions All the experiments involved authentic viruses were performed in BSL-3 labs. All the experiments involved pseudov1ruses were performed in BSL-2 labs

Biosecurity oversight The biosecurity oversight and evaluations of this work has been done by upstream authorities before this work started

Benefits The neutralizing assay performed by authentic viruses can provide accurate neutralizing ability of antibodies evaluation

Communication benefits The antibodies evaluated with authentic viruses in this study can be potential antiviral agents in the clinical treatment 